\title{
Master Equation Analysis of Post Normal Shock Waves of Nitrogen
}

\author{
Jae Gang Kim* \\ Sejong University, Seoul 143-747, Republic of Korea \\ and \\ Iain D. Boyd亡 \\ University of Michigan, Ann Arbor, Michigan 48109 \\ DOI: $10.2514 / 1 . T 4249$
}

\begin{abstract}
One-dimensional post normal shock flow calculations are carried out using state-of-the-art thermochemical nonequilibrium models. Two-temperature, four-temperature, and electronic master equation coupling models are adopted in the present work. In the four-temperature model, the rotational nonequilibrium is described by Parker and modified Park models. In the electronic master equation coupling model, recently evaluated electron and heavyparticle impacts and radiative transition cross-sections are employed in constructing the system of electronic master equations. In analyzing the shock-tube experiments, the results calculated by the state-of-the-art thermochemical nonequilibrium models are compared with existing shock-tube experimental data. The four-temperature and electronic master equation coupling models with rotational nonequilibrium described by the modified Park model approximately reproduce the measured rotational, vibrational, and electronic temperatures.
\end{abstract}

\section{Introduction}

$\mathbf{T}$ HE energy modes contained in atoms or molecules are usually characterized by a temperature. In a thermochemical nonequilibrium gas mixture, the temperatures that characterize these different modes of energy may be different from each other. Twotemperature and multitemperature models [1] are widely used approaches for characterizing these different energy modes. In the two-temperature model, one approximates this situation with two main assumptions. First, one assumes that there are only two different temperatures. The rotational temperature of molecules is assumed to be the same as the translational temperature of heavy particles. Vibrational temperatures of all molecules are assumed to be the same as the electron temperature and the electronic temperature. Second, the forward and reverse rate coefficients for the chemical reactions involving molecules are assumed to be a function of a geometrically averaged temperature evaluated using the translational and vibrational temperatures. In the multitemperature approach, unlike the two-temperature model, the vibrational temperature is considered as a species-dependent characteristic temperature. Computational fluid dynamic results calculated by these temperature models show fairly good agreement with previous hypersonic flight experiments [2]. However, the two-temperature and multitemperature models are not able to correctly predict the shock standoff distance for a sphere at intermediate hypersonic speeds between Mach numbers of 10 to 15 in air. In the work of Furudate et al. [3] , a comparison of the measured and calculated shock standoff distances was performed. The measurement was made in a ballistic range at Tohoku University, Japan. The calculations were made using the extended two-temperature model in which the vibrational temperatures of $\mathrm{N}_{2}, \mathrm{O}_{2}$, and $\mathrm{NO}$ were considered different from each other. In this work, it was shown that the two-temperature model tends to underestimate the shock standoff distance. In reentry calculations in which the velocity exceeds $10 \mathrm{~km} / \mathrm{s}$, significant diffierences are shown between the

Presented as Paper 2013-3150 at the 44th AIAA Thermophysics Conference, San Diego, CA, 24-27 June 2013; received 5 August 2013; revision received 10 November 2014; accepted for publication 7 December 2014; published online 23 March 2015. Copyright $@ 2014$ by the American Institute of Aeronautics and Astronautics, Inc. All rights reserved. Copies of this paper may be made for personal or internal use, on condition that the copier pay the $\$ 10.00$ per-copy fee to the Copyright Clearance Center, Inc., 222 Rosewood Drive, Danvers, MA 01923; include the code 1533-6808/15 and $\$ 10.00$ in correspondence with the CCC.

*Assistant Professor, Department of Aerospace System Engineering; jaemisunkim@gmail.com. Member AIAA.

†Professor, Department of Aerospace Engineering; Iainboyd@umich.edu. Fellow AIAA. results calculated by those thermochemical models and measured data [4]. These disagreements between the calculated and measured data show the uncertanties of two- and multitemperature models and also show that the thermochemical nonequilibrium behind a strong shock wave is not understood well in the intermediate and higher hypersonic speeds.

One of the modeling uncertainties is the rotational nonequilibrium of air species. The spectrum of radiation emitted in the flow behind a shock wave was measured and analyzed by several researchers $[\underline{5}, 6]$. At the point of peak radiation intensity of the $\mathrm{N}_{2}(2+)$ band, the spectrum was analyzed in detail. From such analyses, it was found that the rotational temperature is not higher than the vibrational temperature at the peak-intensity point. In a recent measurement by the coherent anti-Stokes Raman scattering (CARS) method [ $[$,, 8$]$, the temperature of electronic ground state $\mathrm{N}_{2}$ was estimated from radiation of the strong shock wave in a free-piston, double-diaphragm shock tube. In this experiment, the estimated rotational temperature is not higher than the vibrational temperature. In theoretical calculations of the electronic ground state of $\mathrm{N}_{2}$, master equation studies and one-dimensional (1D) post normal shock flow calculations were performed by Kim and Boyd [9] by using a NASA database [10,11] of state-to-state transition rates for $\mathrm{N}_{2}$. In this study, it was recommended that the rotational energy of $\mathrm{N}_{2}$ should be treated as a nonequilibrium mode in hypersonic reentry calculations.

In the present work, 1D post normal shock flow calculations of $\mathrm{N}_{2}$ are carried out to analyze existing shock-tube experiments [5-8] by state-of-the-art thermochemical nonequilibrium models. The two-temperature (2T), four-temperature (4T), and electronic master equation coupling (EM) models are adopted in these post normal shock flow analyses. In the $4 \mathrm{~T}$ model, the rotational, vibrational, and electron-electronic energies are considered as nonequilibrium modes. The rotational nonequilibrium of the 4T model is described by Parker [12] and modified Park models $[\underline{9}, 13]$ in the present work. In the EM model, the nonequilibrium populations of the electronic states of $\mathrm{N}$ and $\mathrm{N}_{2}$ are determined by solving the system of electronic master equations in order to treat the electronic nonequilibrium energy mode. The system of electronic master equations is constructed by recently evaluated electron and heavy-particle impacts and radiative transition cross-sections of $\mathrm{N}$ and $\mathrm{N}_{2}$. In the EM model, this system of electronic master equations is coupled with the $4 \mathrm{~T}$ model with the rotational nonequilibrium of the modified Park model in calculating the post normal shock flows.

\section{Thermochemical Nonequilibrium Model}

In the present work, five species of $\mathrm{N}, \mathrm{N}_{2}, \mathrm{~N}^{+}, \mathrm{N}_{2}^{+}$, and $\mathrm{e}^{-}$are adopted to carry out the post normal shock flow calculations. For the 
neutral species, 82 electronic states of $\mathrm{N}$ and 5 electronic states of $\mathrm{N}_{2}$ are employed, and for the charged species, the electronic ground states of $\mathrm{N}^{+}$and $\mathrm{N}_{2}^{+}$are considered. The spectral data of each electronic states are obtained from the work by Hyun [14].

In calculating the post normal shock flows, the jump conditions immediately behind a shock wave are derived by using the RankineHugoniot relations assuming the electronic, rotational, and vibrational energy modes to be frozen. The downstream flow is calculated by solving the one-dimensional conservation equations of mass, momentum, and global energy. These conservation equations are defined as

$$
\frac{\partial}{\partial x}\left[\begin{array}{c}
\rho_{s} u \\
\rho u^{2}+p \\
\rho u\left(h+\frac{1}{2} u^{2}\right)
\end{array}\right]=\left[\begin{array}{c}
m_{s} \omega_{s} \\
0 \\
-Q_{\mathrm{rad}}
\end{array}\right]
$$

where $s$ is the species index; $\rho$ is the density; $x$ and $u$ are the distances from the shock wave and the downstream velocity, respectively; $h$ is the specific enthalpy; $m_{s}$ is the species mass; and $\omega_{s}$ is the rate of formation of species number density. In the $2 \mathrm{~T}$ and $4 \mathrm{~T}$ models, the radiative energy loss $Q_{\text {rad }}$ is set to zero. However, in the EM model, the radiative energy loss caused by the radiative processes is considered in the global energy conservation equation. In addition to Eq. (1), the thermochemical nonequilibrium equations of the 2T, 4T, and EM models are solved to analyze the 1D post normal shock flows.

\section{A. Two-Temperature Model}

In Fig. 1, a schematic diagram of nonequilibrium energies and temperatures is presented for the $2 \mathrm{~T}$ model. In the $2 \mathrm{~T}$ model, the transrotational energy is treated as equilibrium, and the electronelectronic-vibrational energy (Eeev) is treated as a nonequilibrium mode. In this $2 \mathrm{~T}$ model, the electron-translational energy (ET), electron-rotational energy (ER), and vibrational-translational energy transfers (VT) and energy removal due to chemical reactions are adopted in describing the relaxation of the electron-electronicvibrational energy. Then, the electron-electronic-vibrational energy conservation equation is constructed as

$$
\begin{aligned}
& u \frac{\partial E_{\mathrm{eev}}}{\partial x}=\sum_{s}^{h} N_{a} \gamma_{e} \frac{2 m_{e}}{m_{s}} v_{s} \frac{3}{2} k\left(T-T_{\mathrm{eev}}\right) \\
& \quad+\sum_{s}^{m} N_{a} \gamma_{e} \frac{2 m_{e}}{m_{s}} g_{r, s} v_{s} \frac{3}{2} k\left(T-T_{\mathrm{eev}}\right) \\
& +\sum_{s}^{m}\left[f_{D} \frac{\widetilde{e_{v, s}}(T) N_{a} \gamma_{s}-E_{v, s}}{\tau_{v, s}}\right] \\
& -\Psi_{v} D_{\mathrm{N}_{2}} N_{a} \omega_{D_{h}}-D_{\mathrm{N}_{2}} N_{a} \omega_{D_{e}}-I_{N^{N}} N_{a} \omega_{\mathrm{EI}} \\
& +\Psi_{v} D_{\mathrm{N}_{2}^{+}} N_{a} \omega_{\mathrm{AI}}-\Psi_{v}\left(D_{\mathrm{N}_{2}}-D_{\mathrm{N}_{2}^{+}}\right) N_{a} \omega_{\mathrm{CER}}
\end{aligned}
$$

where $N_{a}$ is Avogadro's number, $\gamma$ is the species concentration, and $k$ is the Boltzmann constant. In the energy loss term of Eq. (2), $D$ and $I$ are the dissociation and ionization energies, respectively. In Eq. (2), $\omega_{D}$ are the dissociation rates by the electron and heavy-particleimpact processes, $\omega_{\mathrm{EI}}$ is the electron-impact ionization rate, $\omega_{\mathrm{AI}}$ is the associative ionization rate, and $\omega_{\mathrm{CER}}$ is the charge exchange reaction rate. In the $2 \mathrm{~T}$ model, all of the chemical reactions are treated by Arrhenius-type rate coefficients with geometrically averaged temperature of the translational and electron-electronic-vibrational temperatures of $T$ and $T_{\text {eev }}$. In the present work, the reaction rate parameters are obtained from the reference data proposed by Park et al. [15]. The equilibrium constants are applied to derive the backward reaction

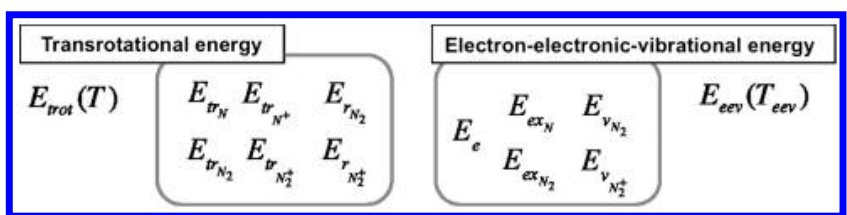

Fig. 1 Schematic diagram of nonequilibrium energies and temperatures in $2 \mathrm{~T}$ model. rates, and these equilibrium constants are calculated by a rigorous method of partition function relations [1]. In the present work, the normalized vibrational energy loss ratio $\bar{\Psi}_{v}$ is set to a constant of 0.5 based on recent master equation studies $[\underline{9}, 16]$ of $\mathrm{N}_{2}$ and $\mathrm{H}_{2}$. In the ET transfer of Eq. (2), the collision frequency $v_{s}$ is calculated as

$$
v_{s}=n_{s} \sigma_{e, s}\left(\frac{8 k T_{\mathrm{eev}}}{\pi m_{e}}\right)^{1 / 2}
$$

where $n_{s}$ is the species number density and $m_{e}$ is the electron mass. In the present work, the effective cross-section $\sigma_{e s s}$ for neutral species is obtained from a curve fit proposed by Gnoffo et al. [17]. For charged species, the effective cross-section with the Debye cutoff approximation [1] is adopted in the present work,

$$
\sigma_{e, s}=\frac{4}{3} \frac{4.39 \times 10^{-6}}{T_{\mathrm{eev}}^{2}} \ln \left(\frac{1.24 \times 10^{4} T_{\mathrm{eev}}^{1.5}}{\sqrt{n_{e}}}\right)
$$

where $n_{e}$ is the electron number density. In the ER transfer of Eq. (2), a model proposed by Nishida and Matsumoto [18] is employed. In the present work, the coefficient $g_{r, s}$ of the ER transfer is set to a constant of 10.0 for neutral and charged particles [18]. In the VT transfer, the Millikan-White relaxation time [19] $\tau_{v, s}$ with the collision-limited correction term [20] $\tau_{c}$ is adopted in the present work. This collisionlimited correction term is defined as

$$
\begin{gathered}
\tau_{c}=\left(n_{t} \sigma_{v} \sqrt{\frac{8 k T}{\pi \mu}}\right)^{-1} \\
\sigma_{v}=\sigma_{v}^{*}\left(\frac{50,000}{T}\right)^{2}
\end{gathered}
$$

where $n_{t}$ and $\mu$ are the total number density of colliding particles and the average mass of the mixture, respectively. In the $2 \mathrm{~T}$ model, $\sigma_{v}^{*}$ is set to $3.0 \times 10^{-17} \mathrm{~cm}^{2}$ as proposed by Park [20]. The diffusion correction factor $f_{D}$ of the VT transfer in Eq. (2) is defined as

$$
f_{D}=\left|\frac{T_{\mathrm{sw}}-T_{\mathrm{eev}}}{T_{\mathrm{sw}}-T_{\mathrm{eev}_{\mathrm{sw}}}}\right|^{a-1}
$$

where $a$ is an arbitrary parameter given as $3.5 \times \exp \left(-5,000 / T_{s}\right)$ for $\mathrm{N}_{2}$ and $\mathrm{N}_{2}^{+} . T_{\mathrm{sw}}$ and $T_{\text {eev }_{\mathrm{sw}}}$ are the translational and electronelectronic vibrational temperatures immediately behind the shock wave.

\section{B. Four-Temperature Model}

In Fig. 2, a schematic diagram of nonequilibrium energies and temperatures is presented for the $4 \mathrm{~T}$ model. In the $4 \mathrm{~T}$ model, four pools of energy are considered, consisting of the translational, rotational, vibrational, and electron-electronic energy modes. In describing rotational nonequilibrium, the Parker [12] and modified Park $[9,13]$ models are taken into account in the present work.

In the Parker [12] model, rotational-translational energy transfer (RT) is treated by a Landau-Teller type of equation with the theoretically calculated RT relaxation time. This relaxation time is derived by using the rigid rotator assumption [12]. Then, the rotational energy

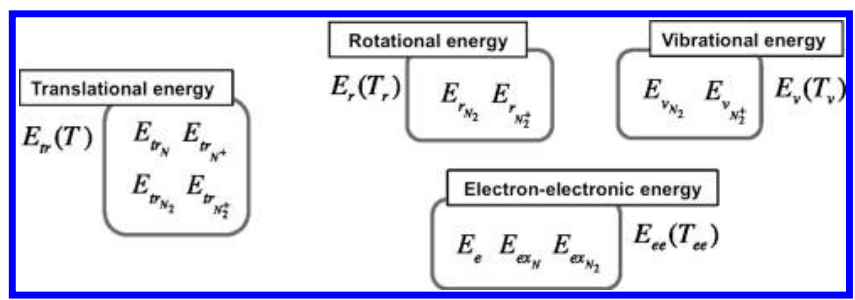

Fig. 2 Schematic diagram of nonequilibrium energies and temperatures in $4 \mathrm{~T}$ model. 
conservation equation is constructed by the ER and RT transfers and the rotational energy losses due to chemical reactions,

$$
\begin{aligned}
u \frac{\mathrm{d} E_{r}}{\mathrm{~d} x} & =-\sum_{s}^{m} N_{a} \gamma_{e} \frac{2 m_{e}}{m_{s}} g_{r, s} v_{s} \frac{3}{2} k\left(T_{r}-T_{\mathrm{ee}}\right) \\
& +\sum_{s}^{m}\left[\frac{\widetilde{e_{r, s}}(T) N_{a} \gamma_{s}-E_{r, s}}{\tau_{r, s}}\right] \\
& -\Psi_{r} D_{\mathrm{N}_{2}} N_{a} \omega_{D}+\Psi_{r} D_{\mathrm{N}_{2}^{+}} N_{a} \omega_{\mathrm{AI}}-\Psi_{r}\left(D_{\mathrm{N}_{2}}-D_{\mathrm{N}_{2}^{+}}\right) N_{a} \omega_{\mathrm{CER}}
\end{aligned}
$$

where $T_{r}$ and $T_{\text {ee }}$ are the rotational and electron-electronic temperatures, respectively. The normalized rotational energy loss ratio $\Psi_{r}$ is set to a constant of 0.3 in the present work. The RT relaxation time $\tau_{r, s}$ of the Parker model is defined as

$$
\tau_{r, s}=Z_{R, s}\left[\sum_{k} \sigma_{\text {rot }} n_{k}\left(\frac{8 k T}{\pi \mu_{s, k}}\right)\right]^{-1}
$$

and $Z_{R, s}$ is given by

$$
Z_{R, s}=\frac{Z_{R, s}^{\infty}}{1+\left(\pi^{3 / 2} / 2\right)\left(\theta_{\mathrm{rot}, s} / T\right)^{1 / 2}+\left(\left(\pi^{2} / 4\right)+\pi\right)\left(\theta_{\mathrm{rot}, s} / T\right)}
$$

where $\theta_{\mathrm{rot}, s}$ is the characteristic rotational temperature. In the present work, the collision cross-section $\sigma_{\text {rot }}$ and number $Z_{R, s}^{\infty}$ are set to $10^{-15} \mathrm{~cm}^{2}$ and 15.7 for $\mathrm{N}_{2}$ and $\mathrm{N}_{2}^{+}$, respectively. In the vibrational energy conservation equation, the electron-vibrational energy (EV) and VT transfers and the vibrational energy losses due to chemical reactions are adopted. This vibrational conservation equation is defined as

$$
\begin{gathered}
u \frac{\partial E_{v}}{\partial x}=\frac{\widetilde{e_{v, \mathrm{~N}_{2}}}\left(T_{\mathrm{ee}}\right) N_{a} \gamma_{s}-E_{v, \mathrm{~N}_{2}}}{\tau_{e V_{\mathrm{N}_{2}}}}+\sum_{s}^{m}\left[f_{D} \frac{\widetilde{e_{v, s}}(T) N_{a} \gamma_{s}-E_{v, s}}{\tau_{v, s}}\right] \\
-\Psi_{v} D_{\mathrm{N}_{2}} N_{a} \omega_{D}+\Psi_{v} D_{\mathrm{N}_{2}^{+}} N_{a} \omega_{\mathrm{AI}}-\Psi_{v}\left(D_{\mathrm{N}_{2}}-D_{\mathrm{N}_{2}^{+}}\right) N_{a} \omega_{\mathrm{CER}}
\end{gathered}
$$

where the $\mathrm{EV}$ relaxation time $\tau_{\mathrm{eV}}$ of $\mathrm{N}_{2}$ is obtained from the measured value by Lee [21] with the curve-fit function of electron-electronic temperature $T_{\mathrm{ee}}$.

In the modified Park model $[9,13]$, the RT relaxation time of $\mathrm{N}_{2}+\mathrm{N}_{2}$ proposed by Park is adopted in the present work. This relaxation time is derived from the results of master equation calculations with the existing state-to-state rotational transition rates. This RT relaxation time is much slower than the relaxation time proposed by Parker [12] at temperatures above 10,000 K. In the rotational-vibrational energy (RV) transfer of $\mathrm{N}_{2}+\mathrm{N}_{2}$, the model of Kim and Boyd [9] is adopted in the present work. In Park's original model [13] of the RV transfer, the fractional contribution of the RV to the total energy transfer is set to a constant of 0.4 , and this value is derived from $f_{\mathrm{RV}}=k T /(k T+1.5 k T)$. In the present work, unlike Park's original model, the fractional contribution for species $s$ is determined by

$$
f_{\mathrm{RV}, s}=\frac{\left(\xi_{v, s} / 2\right) k T}{\left(\xi_{r, s} / 2\right) k T+1.5 k T}
$$

where $\xi_{r, s}$ and $\xi_{v, s}$ are the number of degrees of freedom of the rotational and vibrational modes, respectively. In the modified Park model, the rotational-vibrational-translational energy (RVT) transfer of $\mathrm{N}_{2}(X)+\mathrm{N}\left({ }^{4} S\right)$ is calculated by coupling the full master equations [9] with the rovibrational state-to-state transition rates obtained from the NASA Ames Research Center database $[10,11]$. Then, the ER, EV, RT, VT, RV, and RVT transfers and energy losses due to chemical reactions are employed in constructing the rotational and vibrational energy conservation equations, and these are given as

$$
\begin{aligned}
& u \frac{\mathrm{d} E_{r}}{\mathrm{~d} x}=-\sum_{s}^{m} N_{a} \gamma_{e} \frac{2 m_{e}}{m_{s}} g_{r, s} v_{s} \frac{3}{2} k\left(T_{r}-T_{\mathrm{ee}}\right) \\
& +\sum_{s}^{m}\left[\frac{\widetilde{e_{r, s}}(T) N_{a} \gamma_{s}-E_{r, s}}{\tau_{r, s}}+f_{\mathrm{RV}, s} \frac{E_{v, s}-\widetilde{e_{v, s}}\left(T_{r}\right) N_{a} \gamma_{s}}{\tau_{v, s}}\right] \\
& +\sum_{i=\mathrm{N}_{2}(X, v, J)}^{\mathrm{N}_{2}(X)} \sum_{j=\mathrm{N}_{2}(X, v, J)}^{\mathrm{N}_{2}(X)} e_{r_{\mathrm{N}_{2}}}(i) K(i, j)\left[\frac{Q_{i}}{Q_{j}} \gamma_{j}-\gamma_{i}\right] \rho N_{a}^{2} \gamma_{N\left({ }^{4} S\right)} \\
& -\sum_{i=\mathrm{N}_{2}(X, v, J)}^{\mathrm{N}_{2}(X)} e_{r_{\mathrm{N}_{2}}}(i) K(i, c)\left[\gamma_{i}-\frac{Q_{i} Q_{t_{\mathrm{N}_{2}}}}{Q_{N}^{2} Q_{t_{\mathrm{N}}}^{2}} \exp \left(\frac{D_{i}}{k T}\right) \rho N_{a} \gamma_{N\left({ }^{4} S\right)}^{2}\right] \rho N_{a}^{2} \gamma_{N\left({ }^{4} S\right)} \\
& -\sum_{i=\mathrm{N}_{2}(X, v, J)}^{\mathrm{N}_{2}(X)} e_{r_{\mathrm{N}_{2}}}(i) K_{p}(i, c)\left[\gamma_{i}-\frac{Q_{i} Q_{t_{\mathrm{N}_{2}}}}{Q_{N}^{2} Q_{t_{\mathrm{N}}}^{2}} \exp \left(\frac{D_{i}}{k T}\right) \rho N_{a} \gamma_{N\left({ }^{4} S\right)}^{2}\right] N_{a} \\
& -\Psi_{r} D_{\mathrm{N}_{2}} N_{a} \omega_{D}+\Psi_{r} D_{\mathrm{N}_{2}^{+}} N_{a} \omega_{\mathrm{AI}}-\Psi_{r}\left(D_{\mathrm{N}_{2}}-D_{\mathrm{N}_{2}^{+}}\right) N_{a} \omega_{\mathrm{CER}}
\end{aligned}
$$

$$
\begin{aligned}
& u \frac{\mathrm{d} E_{v}}{\mathrm{~d} x}=\frac{\widetilde{e_{v, \mathrm{~N}_{2}}}\left(T_{\mathrm{ee}}\right) N_{a} \gamma_{s}-E_{v, \mathrm{~N}_{2}}}{\tau_{\mathrm{eV}, \mathrm{N}_{2}}} \\
& +\sum_{s}^{m}\left[\left(1-f_{\mathrm{RV}, s}\right) f_{D} \frac{\widetilde{e_{v, s}}(T) N_{a} \gamma_{s}-E_{v, s}}{\tau_{v, s}}\right. \\
& \left.+f_{\mathrm{RV}, s} \frac{\widetilde{e_{v, s}}\left(T_{r}\right) N_{a} \gamma_{s}-E_{v, s}}{\tau_{v, s}}\right] \\
& +\sum_{i=\mathrm{N}_{2}(X, v, J)}^{\mathrm{N}_{2}(X)} \sum_{j=\mathrm{N}_{2}(X, v, J)}^{\mathrm{N}_{2}(X)} e_{v_{\mathrm{N}_{2}(X)}}(i) K(i, j)\left[\frac{Q_{i}}{Q_{j}} \gamma_{j}-\gamma_{i}\right] \rho N_{a}^{2} \gamma_{N\left({ }^{4} S\right)} \\
& -\sum_{i=\mathrm{N}_{2}(X, v, J)}^{\mathrm{N}_{2}(X)} e_{v_{\mathrm{N}_{2}(X)}}(i) K(i, c) \\
& \times\left[\gamma_{i}-\frac{Q_{i} Q_{t_{\mathrm{N}_{2}}}}{Q_{N}^{2} Q_{t_{\mathrm{N}}}^{2}} \exp \left(\frac{D_{i}}{k T}\right) \rho N_{a} \gamma_{N\left({ }^{4} S\right)}^{2}\right] \rho N_{a}^{2} \gamma_{N\left({ }^{4} S\right)} \\
& -\sum_{i=\mathrm{N}_{2}(X, v, J)}^{\mathrm{N}_{2}(X)} e_{v_{\mathrm{N}_{2}(X)}}(i) K_{p}(i, c)\left[\gamma_{i}-\frac{Q_{i} Q_{t_{\mathrm{N}_{2}}}}{Q_{N}^{2} Q_{t_{\mathrm{N}}}^{2}} \exp \left(\frac{D_{i}}{k T}\right) \rho N_{a} \gamma_{N\left({ }^{4} S\right)}^{2}\right] N_{a} \\
& -\Psi_{v} D_{\mathrm{N}_{2}} N_{a} \omega_{D}+\Psi_{v} D_{\mathrm{N}_{2}^{+}} N_{a} \omega_{\mathrm{AI}}-\Psi_{v}\left(D_{\mathrm{N}_{2}}-D_{\mathrm{N}_{2}^{+}}\right) N_{a} \omega_{\mathrm{CER}}
\end{aligned}
$$

where indices of $i$ and $j$ denote the rovibrational state of $\mathrm{N}_{2}(X)$. The bound-bound and bound-free rates of $K(i, j)$ and $K(i, c)$ are the rovibrational state-to-state transition rates for $\mathrm{N}_{2}(X)+\mathrm{N}\left({ }^{4} S\right)$, respectively. In Eqs. (13) and (14), $Q_{i}$ is the rovibrational partition function of the electronic ground state of $\mathrm{N}_{2}$. In the modified Park model, the predissociation of $\mathrm{N}_{2}(X)$ is included, and its rate is described by $K_{p}(i, c)$. In the previous master equation studies for $\mathrm{N}+\mathrm{N}_{2}[\underline{9}]$ and $\mathrm{H}_{2}$ and He mixtures [16,22,23], it was observed that the rotational and vibrational relaxation times become identical when the temperature increases. In the work by Kim and Boyd [9], the collision-limiting cross-section $\sigma_{v}$ of Eq. (6) was modified to a constant of $3.0 \times 10^{-18} \mathrm{~cm}^{2}$ to satisfy these relaxation patterns. In the modified Park model, this corrected collision-limiting cross-section is employed.

In Eqs. (13) and (14), the unknown variable is the rovibrational concentration $\gamma_{i}$ of electronic ground state $\mathrm{N}_{2}$. Unfortunately, we cannot directly calculate $\gamma_{i}$ from state-to-state kinetics because of the lack of knowledge about the transition rates for $\mathrm{N}_{2}(X)+\mathrm{N}_{2}(X)$. In the present work, the rovibrational concentration $\gamma_{i}$ is determined from the Boltzmann distributions using the nonequilibrium temperatures as

$$
\gamma_{i}=\frac{\eta\left(i, T_{r}, T_{v}\right)}{\sum_{i} \eta\left(i, T_{r}, T_{v}\right)} \gamma_{\mathrm{N}_{2}}
$$

$$
\eta\left(i, T_{r}, T_{v}\right)=\epsilon g_{e} g_{s}(2 J+1) \exp \left[-\frac{e_{r}(i)}{k T_{r}}-\frac{e_{v}(i)}{k T_{v}}\right]
$$


where $J$ is the rotational quantum number, $\epsilon$ is a symmetry factor, and $g_{e}$ and $g_{s}$ are the total degeneracy of the electronic state and the nuclear spin multiplicity of $\mathrm{N}_{2}$, respectively.

In species conservation equations of the modified Park model, all of the chemical reactions are described by using the Arrhenius type of equations except the dissociation of $\mathrm{N}_{2}(X)+\mathrm{N}\left({ }^{4} S\right)$ and the predissociation of $\mathrm{N}_{2}(X)$. These dissociation and predissociation processes are evaluated by coupling the rovibrational master equation of the bound-free transitions. These are given as

$$
\begin{aligned}
& \omega_{D \mid \mathrm{N}_{2}+\mathrm{N}}=\sum_{i=\mathrm{N}_{2}(X, v, J)}^{\mathrm{N}_{2}(X)} K(i, c) \\
& \times\left[\gamma_{i}-\frac{Q_{i} Q_{t_{\mathrm{N}_{2}}}}{Q_{N}^{2} Q_{t_{\mathrm{N}}}^{2}} \exp \left(\frac{D_{i}}{k T}\right) \rho N_{a} \gamma_{N\left({ }^{4} S\right)}^{2}\right] \rho N_{a} \gamma_{N\left({ }^{4} S\right)} \\
& \omega_{D \mid \mathrm{N}_{2}}=\sum_{i=\mathrm{N}_{2}(X, v, J)}^{\mathrm{N}_{2}(X)} K_{p}(i, c)\left[\gamma_{i}-\frac{Q_{i} Q_{t_{\mathrm{N}_{2}}}}{Q_{N}^{2} Q_{t_{\mathrm{N}}}^{2}} \exp \left(\frac{D_{i}}{k T}\right) \rho N_{a} \gamma_{N\left({ }^{4} S\right)}^{2}\right]
\end{aligned}
$$

The electron-electronic energy conservation equation using the Parker and modified Park models is constructed by ET, ER, and EV transfers and is defined as electron-impact excitation, the excitation rates of the first three states of $\mathrm{N}$ are obtained from the work by Bultel et al. [24]. The electronic state-to-state transition rates of the other states are obtained from the work by Panesi et al. [25,26]. In electron-impact ionization, the rate is well known for atomic $\overline{\mathrm{H}}$ [14], and this rate form is adopted for ionization of $\mathrm{N}$ in the present work. This electron-impact ionization rate is defined as

$$
K_{e}(i, c)=\frac{5.45}{T_{e}^{3 / 2}}\left[0.62 \frac{k T_{e}}{I_{N}-E_{i}} E_{1}\left(\frac{I_{N}-E_{i}}{k T_{e}}\right)-0.534 \frac{E_{1}\left(E_{c}\right)}{E_{c}}\right]
$$

where index $i$ denotes the electronic state of $\mathrm{N}$ and $T_{e}$ is the electron temperature. $E_{c}$ is $E_{c}=\left(I_{N}-E_{i}\right) /\left(k T_{e}\right)+0.56$, and $E_{1}(\theta)$ is the exponential integration defined as

$$
E_{1}(\theta)=\int_{0}^{1} \exp (-\theta / s) s^{-1} \mathrm{~d} s
$$

In molecular elementary processes, electron and heavy-particle impact excitation and dissociations are adopted in the EM model. The overall rate of electron-impact excitation is defined as

$$
\bar{K}_{e}\left(e, e^{\prime}\right)=\frac{\sum_{v} \exp \left(-G_{v} / k T_{v}\right) \sum_{J}(2 J+1) \exp \left(-F_{J} / k T_{r}\right) \sum_{v^{\prime}} q\left(v, v^{\prime}\right) \sum_{J^{\prime}}\left(2 J^{\prime}+1\right) K\left(e, v, J ; e^{\prime}, v^{\prime}, J^{\prime}\right)}{\sum_{v} \exp \left(-G_{v} / k T_{v}\right) \sum_{J}(2 J+1) \exp \left(-F_{J} / k T_{r}\right)}
$$

$$
\begin{aligned}
u & \frac{\mathrm{d} E_{\mathrm{ee}}}{\mathrm{d} x}=\sum_{s}^{h} N_{a} \gamma_{e} \frac{2 m_{e}}{m_{s}} v_{s} \frac{3}{2} k\left(T-T_{\mathrm{ee}}\right) \\
& +\sum_{s}^{m} N_{a} \gamma_{e} \frac{2 m_{e}}{m_{s}} g_{r, s} v_{s} \frac{3}{2} k\left(T_{r}-T_{\mathrm{ee}}\right)-\frac{\widetilde{e_{v, N_{2}}}\left(T_{\mathrm{ee}}\right) N_{a} \gamma_{s}-E_{v, \mathrm{~N}_{2}}}{\tau_{\mathrm{eV}, \mathrm{N}_{2}}} \\
& -\left(1-\Psi_{r}-\Psi_{v}\right) D_{\mathrm{N}_{2}} N_{a} \omega_{D_{e}}-I_{N} N_{a} \omega_{\mathrm{EI}}
\end{aligned}
$$

\section{Electronic Master Equation Coupling Model}

In Fig. $\underline{3}$, a schematic diagram of nonequilibrium energies and temperatures is presented for the EM model. In the EM model, the rotational and vibrational energy relaxations are treated in a similar way as the $4 \mathrm{~T}$ with the rotational nonequilibrium of the modified Park model. However, the number density populations of electronic states of $\mathrm{N}$ and $\mathrm{N}_{2}$ are evaluated by solving the system of electronic master equations.

In atomic elementary processes of the system of electronic master equations, the $\mathrm{N}$ atom is more efficiently excited and ionized by electron impact than by the heavy-particle-impact processes. In

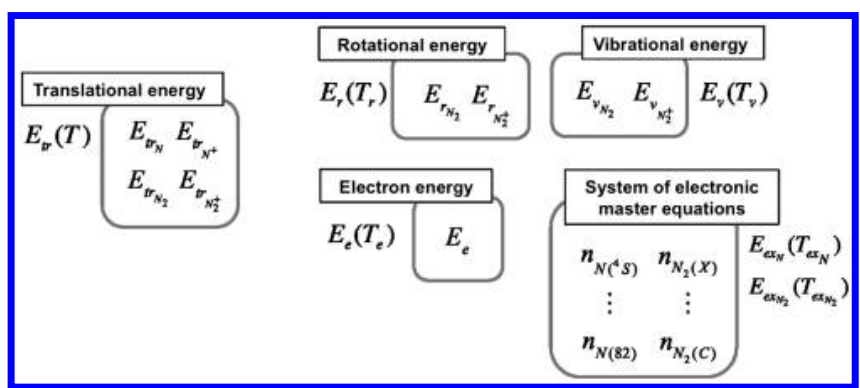

Fig. 3 Schematic diagram of nonequilibrium energies and temperatures in EM model. where $q\left(v, v^{\prime}\right)$ is the Franck-Condon factor. $G_{v}$ and $F_{J}$ are the vibrational and rotational energies, respectively. In Eq. (22), the rate of transition $K_{e}\left(e, v, J ; e^{\prime}, v^{\prime}, J^{\prime}\right)$ is given by

$$
K\left(e, v, J ; e^{\prime}, v^{\prime}, J^{\prime}\right)=\frac{8 \pi}{\sqrt{m_{e}}}\left(\frac{1}{2 \pi k T_{e}}\right)^{3 / 2} \int_{E *}^{\infty} \sigma_{e} \exp \left(-\frac{E}{k T_{e}}\right) E \mathrm{~d} E
$$

where $E^{*}$ is a threshold energy for electronic excitation and $\sigma_{e}$ is the electron-impact excitation cross-section. In the present work, the electron-impact excitation cross-section and Franck-Condon factor are obtained from the work by Hyun [14]. In the EM model, the electron-impact dissociation rates of the molecular elementary processes are given by

$$
K_{e}^{D}(i, c)=A\left(\frac{T_{e}}{6,000}\right)^{n} \exp \left(-\frac{T_{d}}{T_{e}}\right)
$$

where the parameters $A, n$, and $T_{d}$ are obtained from a database of electron-impact transition rates proposed by Park [27]. In the heavyparticle-impact excitation and dissociation, the bound-bound and bound-free transition rates are given as

$$
K_{h}(i, j)=A\left(\frac{T}{6,000}\right)^{n} \exp \left(-\frac{T_{d}}{T}\right)
$$

where the parameters $A, n$, and $T_{d}$ are obtained from a database of heavy-particle-impact transition rates proposed by Park [28].

In radiative transition processes, the radiative transition model for $\mathrm{N}$ is obtained by grouping elementary levels having similar characteristics. The equivalent spontaneous emission probability of each average level is determined based on the detailed emission probabilities from the work by Hyun [14]. In the present work, 
radiative and dielectric recombination is adopted in describing the recombination of $\mathrm{N}^{+}$and $\mathrm{e}^{-}$, and the rate coefficients are obtained from the work by Bourdon and Vervisch [29]. In the radiative transitions for $\mathrm{N}_{2}$, the radiative transition probability from the electronic state $i$ to state $j<i$ is expressed as

$$
A(i, j)=\frac{\sum_{v} A_{v}(i, j) \exp \left(-G_{v} / k T_{v}\right)}{\sum_{v} \exp \left(-G_{v} / k T_{v}\right)}
$$

where the transition probabilities $A_{v}(i, j)$ are obtained from the work by Hyun [14]. In the present work, the possible reabsorption of the emitted radiation is estimated by using the escape factors $\alpha$ without solving the radiative transport equations. It is assumed that an optically thin medium is associated with an escape factor of 1 , whereas for an optically thick medium, the escape factor is set to zero.

The system of the electronic master equation of $\mathrm{N}$ and $\mathrm{N}_{2}$ can be constructed by the electron and heavy-particle impact and radiative transitions, and the rates of the electronic concentration of $\mathrm{N}$ can be written as

$$
\begin{aligned}
& u \frac{\partial \gamma_{i}}{\partial x}=\sum_{j}^{N} K_{e}(i, j) \rho N_{a} \gamma_{e}\left[\frac{Q_{i}^{e}}{Q_{j}^{e}} \gamma_{j}-\gamma_{i}\right] \\
& +K_{e}(i, c) \rho N_{a} \gamma_{e}\left[\frac{Q_{i}^{e} Q_{t_{\mathrm{N}}}^{e}}{Q_{\mathrm{N}^{+}}^{e} Q_{t_{\mathrm{N}^{+}}^{e}}^{e} Q_{e} Q_{t_{e}}^{e}} \exp \left(\frac{I_{\mathrm{N}}}{k T_{e}}\right) \rho N_{a} \gamma_{\mathrm{N}^{+}} \gamma_{e}-\gamma_{i}\right] \\
& -\sum_{j}^{\mathrm{N}_{2}} \delta_{i, k} \varepsilon_{k, l} K_{e}^{D}(j, c) \rho N_{a} \gamma_{e} \\
& \times\left[\frac{Q_{j}^{e} Q_{t_{\mathrm{N}_{2}}}^{e}}{Q_{N(k)}^{e} Q_{N(l)}^{e}\left(Q_{t_{\mathrm{N}}}^{e}\right)^{2}} \exp \left(\frac{D_{j}}{k T_{e}}\right) \rho N_{a} \gamma_{N(k)} \gamma_{N(l)}-\gamma_{\mathrm{N}_{2}(j)}\right] \\
& -\sum_{j}^{\mathrm{N}_{2}} \sum_{s}^{h} \delta_{i, k} \varepsilon_{k, l} K_{h, s}^{D}(j, c) \rho N_{a} \gamma_{s} \\
& \times\left[\frac{Q_{j}^{h} Q_{t_{\mathrm{N}_{2}}}^{h}}{Q_{N(k)}^{h} Q_{N(l)}^{h}\left(Q_{t_{\mathrm{N}}}^{h}\right)^{2}} \exp \left(\frac{D_{j}}{k T}\right) \rho N_{a} \gamma_{N(k)} \gamma_{N(l)}-\gamma_{\mathrm{N}_{2}(j)}\right] \\
& -2 \delta_{i, 1} \sum_{j=\mathrm{N}_{2}(X, v, J)}^{\mathrm{N}_{2}(X)} K_{(j, c)}^{\mathrm{N}_{2}(X)+N\left({ }^{4} S\right)} \\
& \times\left[\frac{Q_{j}^{h} Q_{t_{\mathrm{N}_{2}}}^{h}}{\left(Q_{N\left({ }^{4} S\right)}^{h} Q_{t_{\mathrm{N}}}^{h}\right)^{2}} \exp \left(\frac{D_{j}}{k T}\right) \rho N_{a} \gamma_{\mathrm{N}\left({ }^{4} S\right)}^{2}-\gamma_{j}\right] \rho N_{a} \gamma_{\mathrm{N}\left({ }^{4} S\right)} \\
& -2 \delta_{i, 1} \sum_{j=\mathrm{N}_{2}(X, v, J)}^{\mathrm{N}_{2}(X)} K_{p}^{\mathrm{N}_{2}(X)}(j, c) \\
& \times\left[\frac{Q_{j}^{h} Q_{t_{\mathrm{N}_{2}}}^{h}}{\left(Q_{\mathrm{N}\left({ }^{4} S\right)}^{h} Q_{t_{\mathrm{N}}}^{h}\right)^{2}} \exp \left(\frac{D_{j}}{k T}\right) \rho N_{a} \gamma_{\mathrm{N}\left({ }^{4} S\right)}^{2}-\gamma_{j}\right] \\
& +\sum_{j, j>i}^{N} \alpha_{j, i} A(j, i) \gamma_{j}-\sum_{j, j<i}^{N} \alpha_{i, j} A(i, j) \gamma_{i}+\left[\alpha_{i}^{\mathrm{RR}} K_{\mathrm{RR}}(c, i)\right. \\
& \left.+\alpha_{i}^{\mathrm{DR}} K_{\mathrm{DR}}(c, i)\right] \rho N_{a} \gamma_{\mathrm{N}^{+}} \gamma_{e}
\end{aligned}
$$

where $k$ and $l$ denote the electronic states of the dissociated $\mathrm{N}$ atom. The Dirac delta function is $\delta$, and $\epsilon$ is the symmetric factor defined as $\epsilon_{k, l}=2$ if $k=l$ or $\epsilon_{k, l}=1$ if $k \neq l . K_{e}$ is the electronic-impact excitation rate. $K_{e}^{D}$ and $K_{h}^{D}$ are the electron- and heavy-particleimpact dissociation rates. $K_{\mathrm{RR}}$ and $K_{\mathrm{DR}}$ are the radiative and dielectric recombination rates, respectively. In Eq. (27), $Q^{e}$ is the partition function defined by the electron temperature $T_{e}$, and $Q^{h}$ is defined by the translational temperature $T$. The rates of change of the electronic concentration of $\mathrm{N}_{2}$ are expressed as

$$
\begin{aligned}
& u \frac{\partial \gamma_{i}}{\partial x}=\sum_{j}^{\mathrm{N}_{2}} K_{e}(i, j) \rho N_{a} \gamma_{e}\left[\frac{Q_{i}^{e}}{Q_{j}^{e}} \gamma_{j}-\gamma_{i}\right] \\
&+K_{e}^{D}(i, c) \rho N_{a} \gamma_{e}\left[\frac{Q_{i}^{e} Q_{\mathrm{N}_{2}}^{e}}{Q_{N(k)}^{e} Q_{N(l)}^{e}\left(Q_{t_{\mathrm{N}}}^{e}\right)^{2}} \exp \left(\frac{D_{i}}{k T_{e}}\right) \rho N_{a} \gamma_{N(k)} \gamma_{N(l)}-\gamma_{i}\right] \\
&+\sum_{s}^{h} \sum_{j}^{N_{2}} K_{h, s}(i, j) \rho N_{a} \gamma_{s}\left[\frac{Q_{i}^{h}}{Q_{j}^{h}} \gamma_{j}-\gamma_{i}\right] \\
&+\sum_{s}^{h} K_{h, s}^{D}(i, c) \rho N_{a} \gamma_{s} \\
& \quad \times\left[\frac{Q_{i}^{h} Q_{\mathrm{N}_{2}}^{h}}{Q_{N(k)}^{h} Q_{N(l)}^{h}\left(Q_{t_{\mathrm{N}}}^{h}\right)^{2}} \exp \left(\frac{D_{i}}{k T}\right) \rho N_{a} \gamma_{N(k)} \gamma_{N(l)}-\gamma_{i}\right] \\
&+ \delta_{i, 1} \sum_{j=(v, J)}^{\mathrm{N}_{2}(X)} K_{(j, c)}^{\mathrm{N}_{2}(X)+N\left({ }^{4} S\right)} \\
& \times\left[\frac{Q_{j}^{h} Q_{\mathrm{N}_{2}}^{h}}{\left(Q_{N\left({ }^{4} S\right)}^{h} Q_{t_{\mathrm{N}}}^{h}\right)^{2}} \exp \left(\frac{D_{j}}{k T}\right) \rho N_{a} \gamma_{N\left({ }^{4} S\right)}^{2}-\gamma_{j}\right] \rho N_{a} \gamma_{N\left({ }^{4} S\right)} \\
&+ \delta_{i, 1} \sum_{j=(v, J)}^{\mathrm{N}_{2}(X)} K_{p}^{\mathrm{N}_{2}(X)}(j, c)\left[\frac{Q_{j}^{h} Q_{\mathrm{N}_{2}}^{h}}{\left(Q_{N\left({ }^{4} S\right)}^{h} Q_{t_{\mathrm{N}}}^{h}\right)^{2}} \exp \left(\frac{D_{j}}{k T}\right) \rho N_{a} \gamma_{N\left({ }^{4} S\right)}^{2}-\gamma_{j}\right] \\
&+ \sum_{j, j>i}^{\mathrm{N}_{2}} \alpha_{j, i} A_{\mathrm{N}_{2}}(j, i) \gamma_{j}-\sum_{j, j<i}^{\mathrm{N}_{2}} \alpha_{i, j} A_{\mathrm{N}_{2}}(i, j) \gamma_{i}
\end{aligned}
$$

In the EM model, the electron energy loss and gain due to electronimpact processes of the bound-bound and bound-free transitions need to be considered. In the present work, these detailed electron energy processes are fully implemented in the electron energy conservation equation of Eq. (19) with some modifications. In the EM model, electron energy conservation due to the electron-impact processes is ensured using a bin-counting method for each electronimpact process. For example, for electron-impact bound-bound transitions of $\mathrm{N}$, the electronic excitation of $\mathrm{N}$ is carried out by obtaining the threshold energy from the electron impact. Then, this threshold energy is removed from the electron translational energy. Also, in the EM model, the rotational and vibrational energy loss and gain due to chemical reactions of Eqs. (13) and (14) are modified to describe the chemical reactions in each electronic state. In the EM model, the associative ionization and charge exchange reaction are considered by the Arrhenius type of equations with an assumption that these reactions occur in the electronic ground state. The radiative energy loss $Q_{\text {rad }}$ due to radiative transition and radiative recombination of Eq. (1) are modeled as

$$
\begin{aligned}
Q_{\mathrm{rad}} & =\sum_{s}^{\mathrm{N}, \mathrm{N}_{2}} \sum_{i, j, j<i} \alpha_{i, j}\left(E_{i}-E_{j}\right) A_{s}(i, j) N_{a} \gamma_{i} \\
& +\sum_{i}^{\mathrm{N}}\left(I_{N}-E_{i}\right)\left[\alpha_{i}^{\mathrm{RR}} K_{\mathrm{RR}}(c, i)+\alpha_{i}^{\mathrm{DR}} K_{\mathrm{DR}}(c, i)\right] \rho N_{a}^{2} \gamma_{N^{+}} \gamma_{e}
\end{aligned}
$$

\section{Post Normal Shock Flow Calculations}

The post normal shock flow calculations are carried out for existing shock-tube experiments [5-8] by the state-of-the-art thermochemical nonequilibrium $2 \mathrm{~T}, 4 \overline{\mathrm{T}}$, and EM models. The results from the calculations are compared with the shock-tube measured data and analyzed in detail. In the present work, the post normal shock flow calculations are performed by an implicit integration method with parallelization algorithm to reduce the computation time.

In Fig. 4, the calculated translational, rotational, and vibrational temperatures by the $2 \mathrm{~T}, 4 \mathrm{~T}$, and EM models are compared with the shock-tube data measured by Sharma and Gillespie [5] and AVCO Everett Research Laboratory, Everett, MA [30], in the optically thin medium. In these calculations, the upstream pressure is 1.0 torr, 


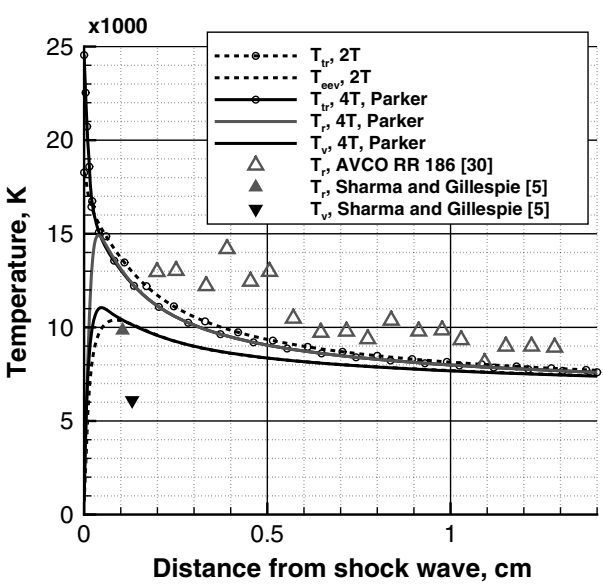

a) $2 \mathrm{~T}$ and $4 \mathrm{~T}$ with Parker models

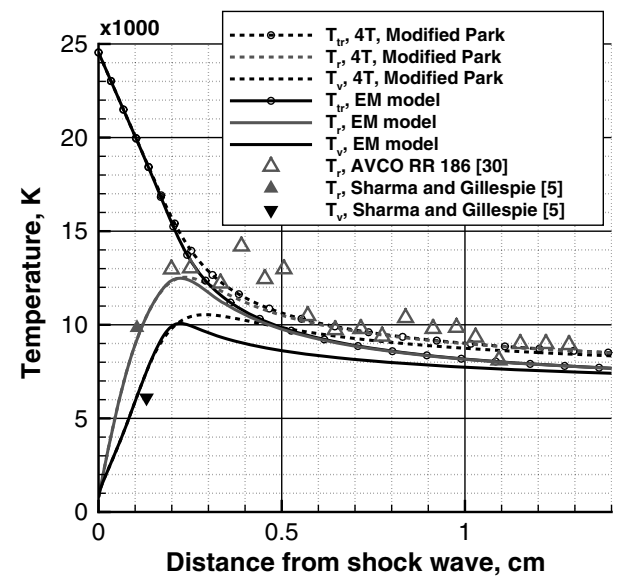

b) 4T with modified Park and EM models

Fig. 4 Comparisons of the calculated translational, rotational, and vibrational temperatures with the shock-tube data measured by Sharma and Gillespie [5] and AVCO [30].

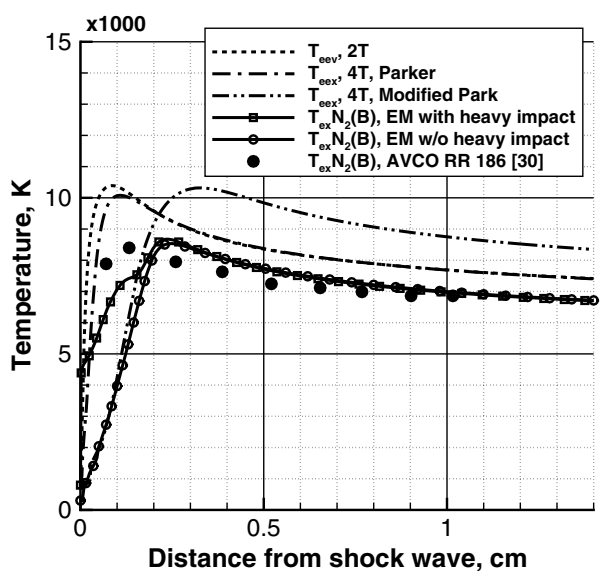

Fig. 5 Comparisons of calculated electronic temperatures of $\mathrm{N}_{2}\left(B^{3} \Pi_{g}\right)$ with the shock-tube data measured by AVCO [30].

and the shock velocity is $6.2 \mathrm{~km} / \mathrm{s}$. In this case, the total enthalpy of the freestream is about $20 \mathrm{MJ} / \mathrm{kg}$, and the density is $1.497 \times 10^{-6} \mathrm{~g} / \mathrm{cm}^{3}$. In Fig. 4 a , the calculated results by the $2 \mathrm{~T}$ and $4 \mathrm{~T}$ with Parker models are compared with the experimental values. The rotational and vibrational relaxations between the $2 \mathrm{~T}$ and $4 \mathrm{~T}$ models are almost identical, and the rotational relaxation of the $4 \mathrm{~T}$ model is fast enough to treat as an equilibrium temperature. In comparison with the experimental values, the calculated relaxations of the rotational and vibrational temperatures are much faster than

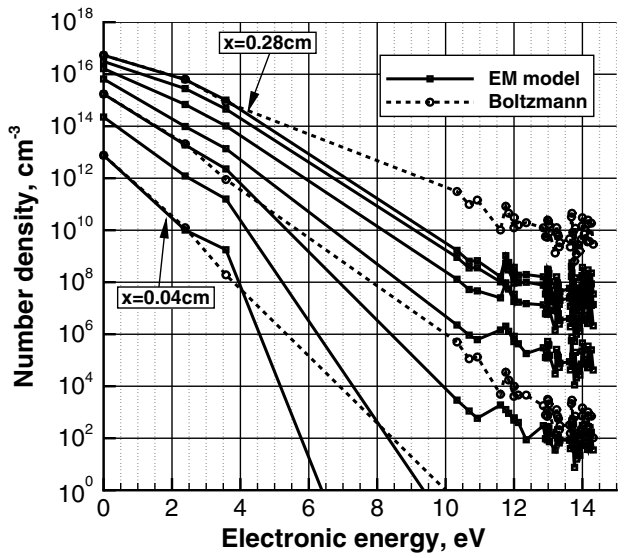

a) Electronic number density populations of $\mathbf{N}$ the measured values by Sharma and Gillespie, and the rotational temperature underestimates the measured rotational temperature by AVCO. In Fig. 4b, the calculated temperatures by the $4 \mathrm{~T}$ with modified Park and EM models are compared with the shock-tube measured values. The calculated rotational relaxation of the $4 \mathrm{~T}$ with modified Park model is slow enough to treat as a nonequilibrium state, and the calculated rotational and vibrational temperatures agree with the data measured by Sharma and Gillespie. In the downstream region, the rotational temperature of the EM model is slightly lower than the 4T with modified Park model. This is because the dependence on the dissociation rates of the excited states of the EM model were determined by an empirical manner due to the lack of knowledge of these processes. In the EM model, the effects of electron- and heavy-particle-impact dissociation on the electronic excited states of $\mathrm{N}_{2}$ are obtained from the work by Park $[27,28]$. Thus, uncertainty in the modeling of these processes affects the rovibrational relaxation of $\mathrm{N}_{2}$. However, the calculated rotational temperature of the 4T with modified Park and EM models agree with the data measured by AVCO.

In Fig. 5 , the calculated electronic temperatures of $\mathrm{N}_{2}\left(B^{3} \Pi_{g}\right)$ by the $2 \mathrm{~T}, 4 \mathrm{~T}$, and EM models are compared with the shock-tube data measured by AVCO [30]. In the EM model, the electronic excitations with and without heavy-particle-impact processes are also compared. In the 2T and 4T with Parker models, the estimated electronic temperatures of $T_{\text {eev }}$ and $T_{\text {eex }}$ are higher than the measured electronic temperature of $\mathrm{N}_{2}\left(B^{3} \Pi_{g}\right)$ by AVCO. In the $4 \mathrm{~T}$ with modified Park model, the estimated electronic temperature of $T_{\text {eex }}$ is also higher than the measured value, and it cannot accurately reproduce the electronic temperature of $\mathrm{N}_{2}\left(B^{3} \Pi_{g}\right)$ immediately behind the shock

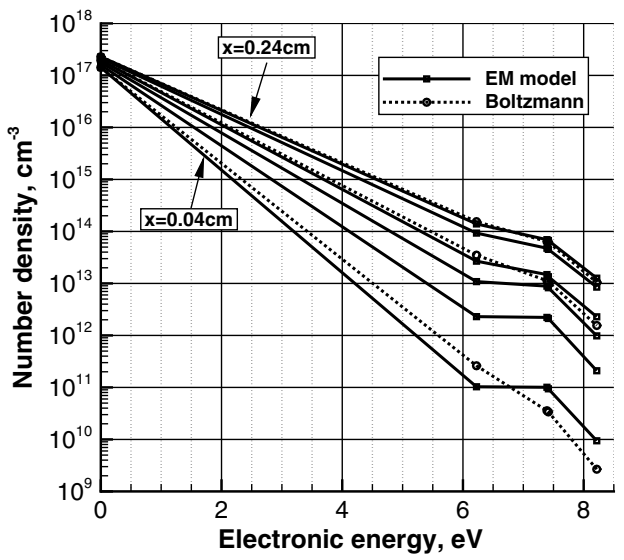

b) Electronic number density populations of $\mathbf{N}_{2}$

Fig. 6 Comparisons of the electronic number density populations of the EM model and the Boltzmann distributions specified by the electron temperature. 


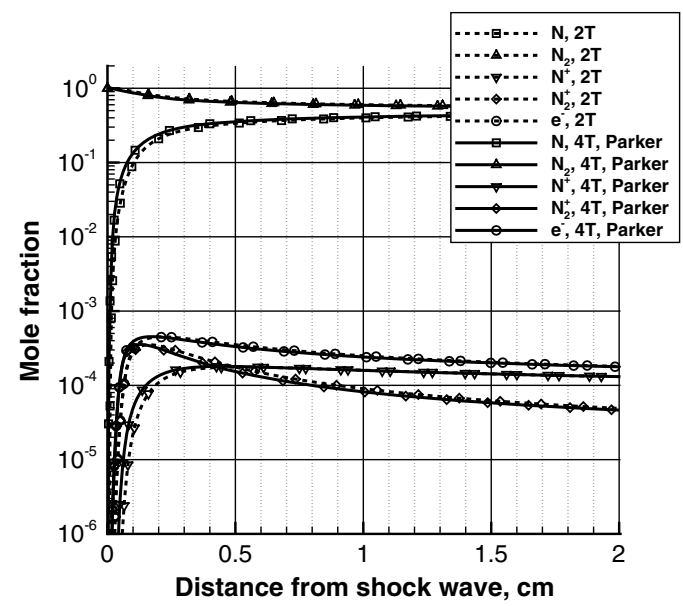

a) $2 \mathrm{~T}$ and $4 \mathrm{~T}$ with Parker models

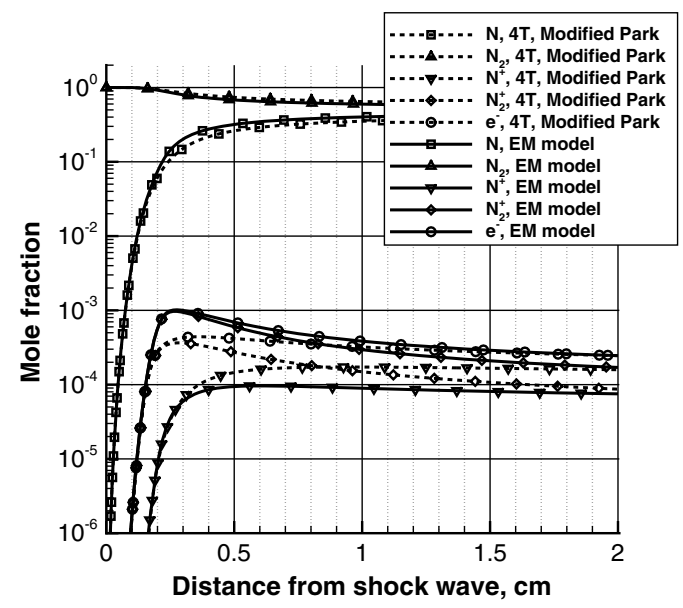

b) 4T with modified Park and EM models

Fig. 7 Comparisons of the species mole fractions in the case of the shock-tube experiments by Sharma and Gillespie [5] and AVCO [30].

wave. In comparisons of the electronic temperature between the EM model without the heavy-particle-impact processes and the experimental values, it is observed that the EM model is unable to accurately describe the electronic excitation immediately behind the shock wave. This is because the number density of electrons is too low in this region to excite the electronic energy levels of $\mathrm{N}_{2}$. In the EM model with the heavy-particle-impact processes, the calculated

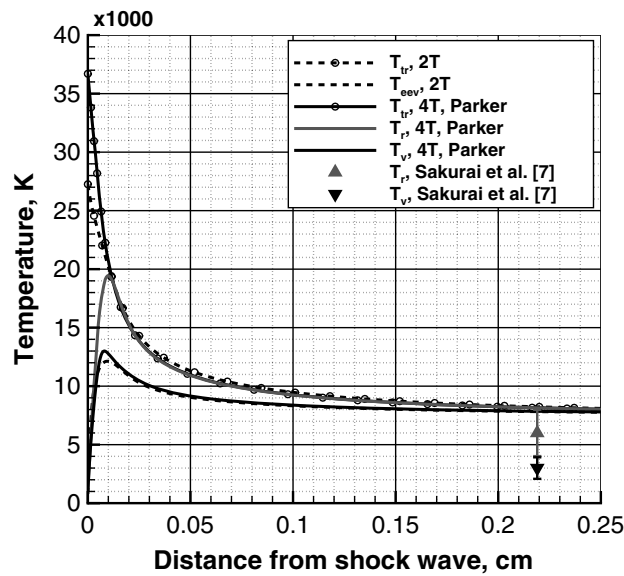

a) $2 \mathrm{~T}$ and $4 \mathrm{~T}$ with Parker models $\left(p_{\infty}=2.5\right.$ torr and $\left.u_{\infty}=7.6 \mathrm{~km} / \mathrm{s}\right)$



c) $2 \mathrm{~T}$ and $4 \mathrm{~T}$ with Parker models

$\left(p_{\infty}=\mathbf{1 0 . 0}\right.$ torr and $\left.u_{\infty}=5.8 \mathrm{~km} / \mathrm{s}\right)$ electronic temperature of $\mathrm{N}_{2}\left(B^{3} \Pi_{g}\right)$ agrees better than the other models. It shows that, immediately behind a shock wave, most of the electronic excitation of $\mathrm{N}_{2}$ occurs through the heavy-particle-impact processes. After the electrons are generated by associative ionization, the dominant electron processes become important in the electronic excitation of $\mathrm{N}_{2}$. However, differences of the electronic temperature between the EM model with heavy-particle-impact processes and

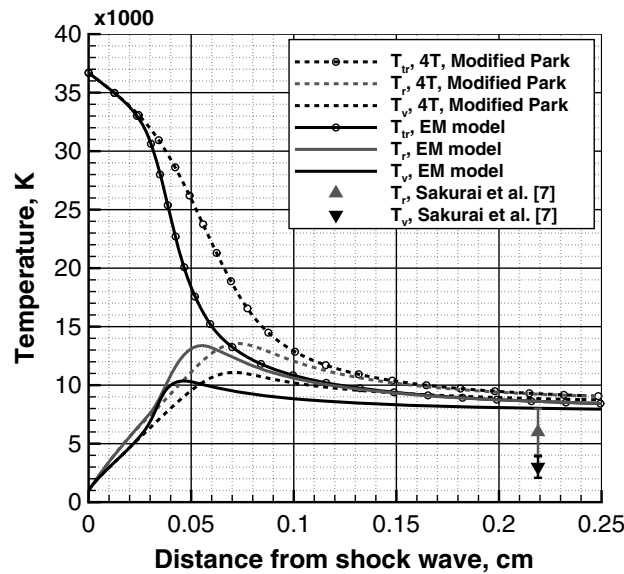

b) 4T modified Park and EM models

$\left(p_{\infty}=2.5\right.$ torr and $u_{\infty}=7.6 \mathrm{~km} / \mathrm{s}$ )

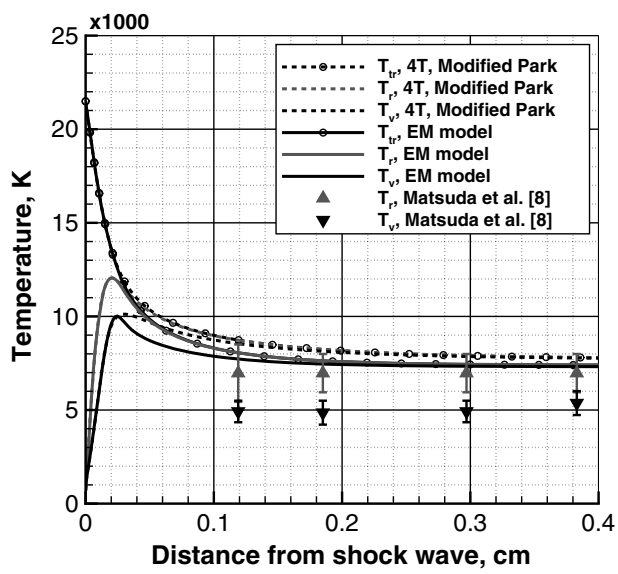

d) 4T modified Park and EM models

( $p_{\infty}=10.0$ torr and $u_{\infty}=5.8 \mathrm{~km} / \mathrm{s}$ )

Fig. 8 Comparisons of the calculated translational, rotational, and vibrational temperatures with the shock-tube measured values by CARS method $[7,8]$. 


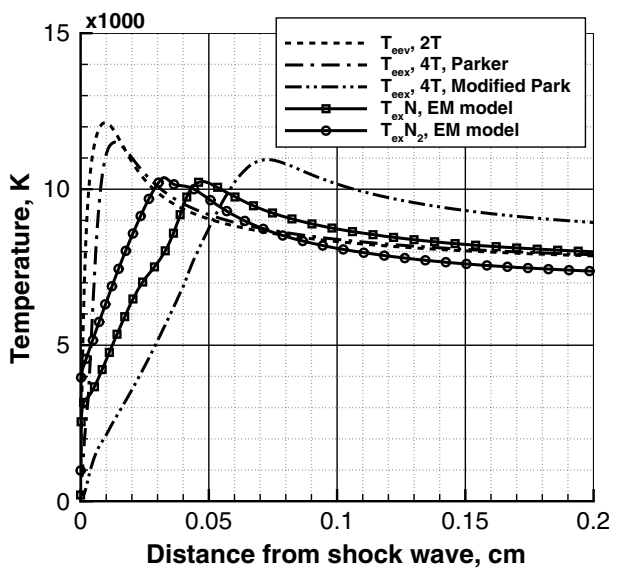

Fig. 9 Comparisons of the electronic temperatures for the 2T, 4T with Parker, 4T with modified Park, and EM models in the case of the shocktube experiment by Sakurai et al. [7].

the measured values still exist and are most likely due to a lack of accuracy in the cross-sections for the heavy-particle-impact excitation.

In Fig. 6, the electronic nonequilibrium populations of $\mathrm{N}$ and $\mathrm{N}_{2}$ of the EM model are compared with those of the Boltzmann distributions specified by the electron temperature. In Fig. 6a, the nonequilibrium populations of $\mathrm{N}$ are presented. Immediately behind the shock wave, large deviations of the number density between the EM model and the Boltzmann distributions are observed in the highly excited states. The populations of the low electronic states can be treated as Boltzmann distributions specified by electron temperature. However, the nonequilibrium populations of the highly excited states are not converged to the Boltzmann distributions, and the degree of nonequilibrium does not decrease uniformly as the flow goes downstream because of the electronic quasi-steady state established through the coupled electronic transitions of electron-impact excitation and ionization [1]. This phenomenon is typical of the nonequilibrium conditions encountered during a high-speed shock velocity [25]. In Fig. 6b, the number density populations of the electronic states of $\mathrm{N}_{2}$ from the EM model are compared with the Boltzmann distributions. Weak deviations between the nonequilibrium populations and the Boltzmann distributions are observed behind the shock wave, and these nonequilibrium populations are almost converged to the Boltzmann distributions in the downstream.

In Fig. 7, the species mole fractions of the 2T, 4T, and EM models are compared. In Figs. $7 \mathrm{a}$ and $7 \mathrm{~b}$, it is observed that the chemical reactions by the $2 \mathrm{~T}$ and $4 \mathrm{~T}$ with Parker models occur more rapidly than the chemical reactions by the 4T with modified Park and EM models. This is because, immediately behind the shock wave, the

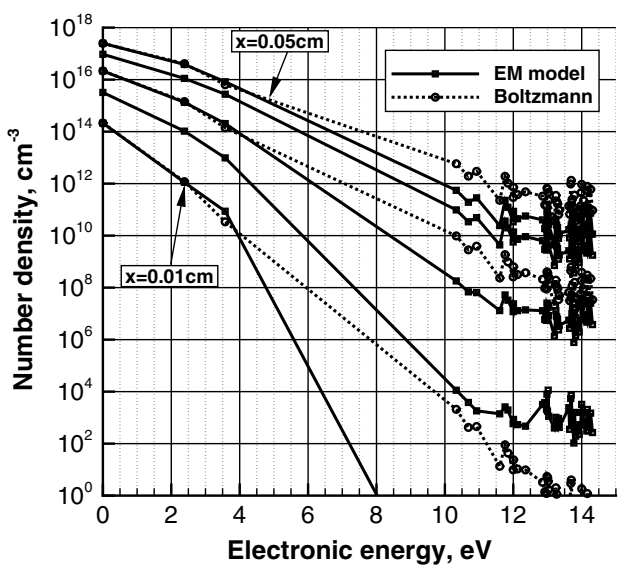

a) Electronic number density populations of $\mathrm{N}$ heavy-particle-impact dissociation has an important role in chemical reactions, and this dissociation is dominated by the rotational and vibrational relaxations. As seen in Fig. 1, such relaxations of the $2 \mathrm{~T}$ and 4T with Parker models are much faster than those of the 4T with modified Park and EM models. When the dissociated $\mathrm{N}$ atom is generated, the electron is easily produced by associative ionization. Then, the electron-impact processes and the other chemical reactions occur. These results show that the heavy-particle-impact rotational and vibrational relaxations immediately behind the shock wave have an important role in the nonequilibrium chemical reactions in post normal shock flows.

In Fig. 8, comparisons of the calculated translational, rotational, vibrational, and electronic temperatures with the shock-tube experiments $[\underline{7}, 8]$ are presented in an optically thin medium. In these shocktube experiments, the CARS method was employed to measure the temperatures of the ground state from the radiation behind the strong shock wave. ND:YAG and dye lasers were used in this method. When these lasers excited the $\mathrm{N}$ molecules behind the shock wave, the CARS signal was collected by a spectrograph. Then, the rotational and vibrational temperatures were estimated using a spectral matching method. In these shock-tube experiments by CARS $[7,8]$, the rotational and vibrational temperatures were measured at the shock wave velocities of 7.6 and $5.9 \mathrm{~km} / \mathrm{s}$ and ambient pressures of 2.5 and 10.0 torr, respectively. In the present work, the post normal shock flow calculations are performed for these conditions. In Figs. 8a-8d, it is observed that the calculated rotational and vibrational temperatures of the 4T with modified Park and EM models increase more slowly behind the shock wave than those of the 2T and 4T with Parker models. Also, the rotational and vibrational relaxations are almost identical in the 4T with modified Park and EM models. In comparing with the measured values, all of the calculated rotational and vibrational temperatures overestimate the experimental values, even though the measured rotational and vibrational temperatures are not identical in the equilibrium positions. However, in the CARS analysis work by Matsuda et al. [8] , it is observed that the theoretical CARS spectrum was calculated based on a simple model without the pressure effect, and the measured data could be improved by improving the spectral model.

In Fig. 9, comparisons of the electronic temperatures of $\mathrm{N}$ and $\mathrm{N}_{2}$ are presented. The estimated electronic temperatures of the $2 \mathrm{~T}$ and $4 \mathrm{~T}$ with Parker models rapidly increase because of the ET and EV transfers of $\mathrm{N}_{2}$. However, the electronic temperatures of the $4 \mathrm{~T}$ with modified Park and EM models slowly increase because the vibrational relaxation occurs more slowly than the $2 \mathrm{~T}$ and $4 \mathrm{~T}$ with Parker models. Also, in the EM model, it is shown that the heavyparticle-impact processes efficiently affect the electronic excitation of $\mathrm{N}_{2}$ behind the shock wave.

In Fig. 10, the nonequilibrium populations of $\mathrm{N}$ and $\mathrm{N}_{2}$ of the EM model are compared with those of the Boltzmann distributions specified by the electron temperature. In Fig. 10a, it is shown that the

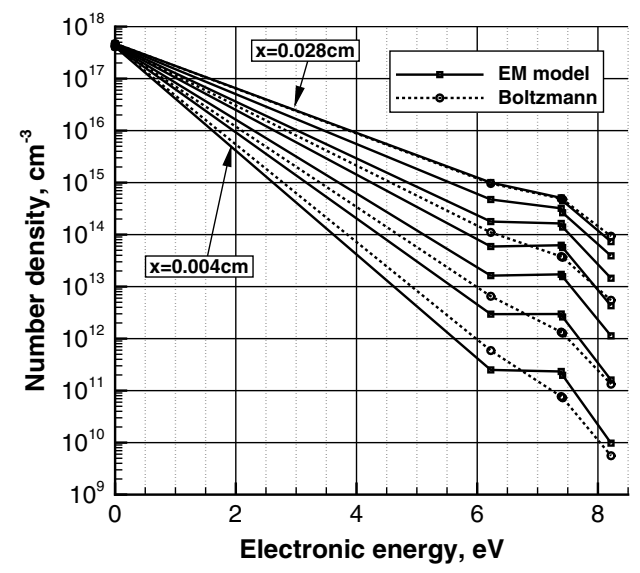

b) Electronic number density populations of $\mathbf{N}_{2}$

Fig. 10 Comparisons of the electronic number density populations of the EM model and the Boltzmann disributions specified by electron temperatures in the case of the shock-tube experiment by Sakurai et al. [7]. 


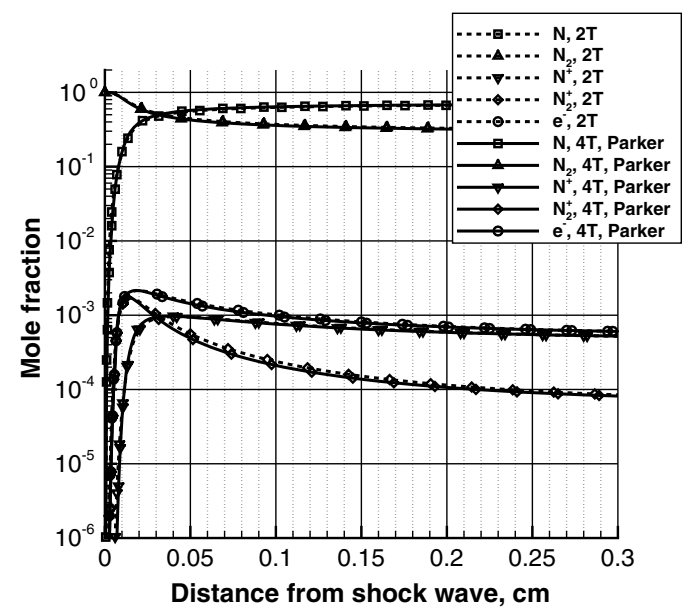

a) $2 \mathrm{~T}$ and $4 \mathrm{~T}$ with Parker models

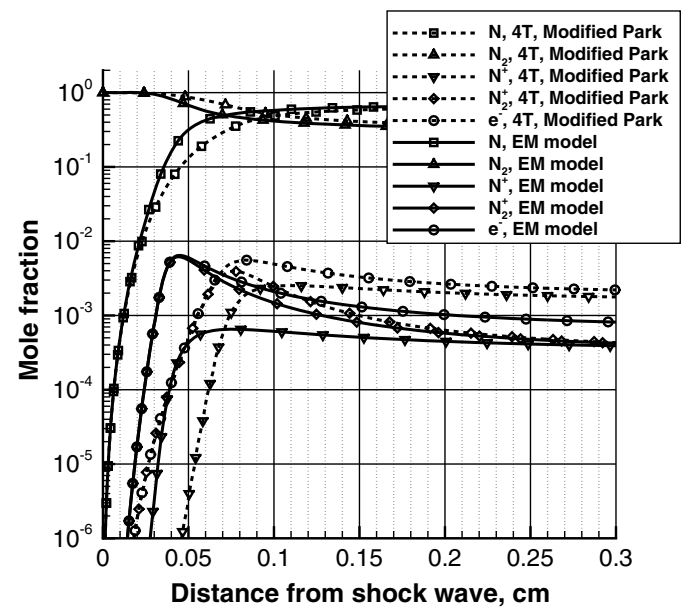

b) 4T with modified Park and EM models

Fig. 11 Comparisons of the species mole fractions in the case of the shock-tube experiment by Sakurai et al. [7].

nonequilibrium populations at low electronic energy of $\mathrm{N}$ are enough to treat as the Boltzmann distributions specified by electron temperature. However, the nonequilibrium populations of the highly excited states are not converged to the Boltzmann distributions. In Fig. 10b, the number density populations of the EM model and the Boltzmann distributions are compared for the electronic states of $\mathrm{N}_{2}$. Immediately behind the shock wave, weak deviations between the nonequilibrium populations and the Boltzmann distributions are observed, and the nonequilibrium populations are almost converged to the Boltzmann distributions specified by the electron temperature in the downstream.

In Fig. 11, comparisons of the species mole fractions for the 2T, 4T, and EM models are presented. In Figs. $11 \mathrm{a}$ and $11 \mathrm{~b}$, it is observed that the chemical reactions by the $2 \mathrm{~T}$ and $\overline{4 \mathrm{~T}}$ with $\overline{\text { Parker models occur }}$ more rapidly than the chemical reactions by the $4 \mathrm{~T}$ with modified Park and EM models because the rotational and vibrational relaxations of the 2T and 4T with Parker models are much faster than the 4T with modified Park and EM models. These rotational and vibrational

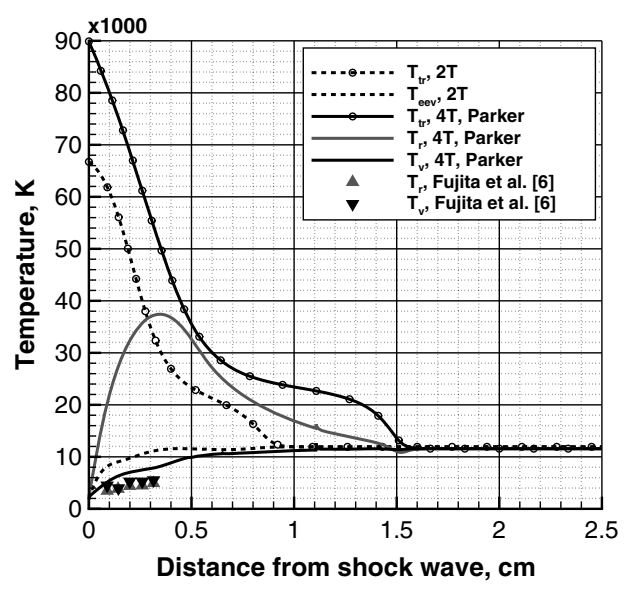

a) $2 \mathrm{~T}$ and $4 \mathrm{~T}$ with Parker models

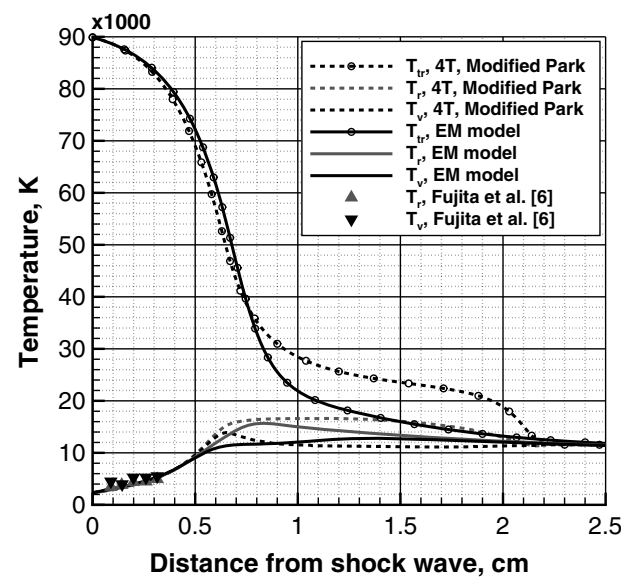

b) $4 \mathrm{~T}$ with modified Park and EM models

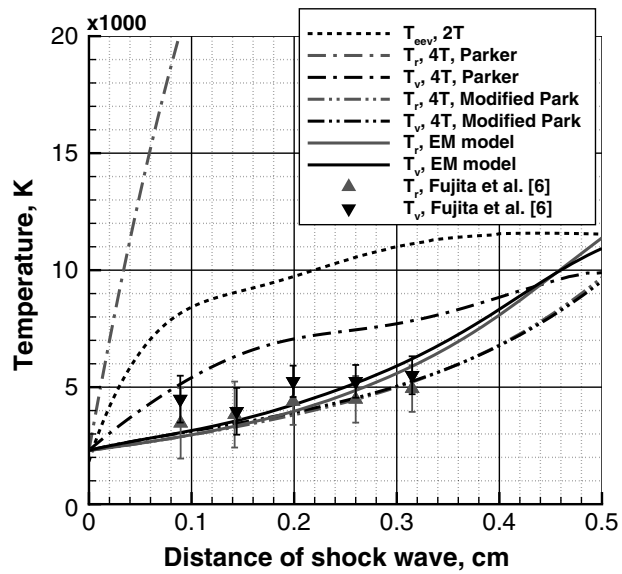

c) Comparisons between the calculated and measured temperatures

Fig. 12 Comparisions of the calculated translational, rotational, vibrational temperatures with the shock-tube data measured by Fujita et al. [6]. 


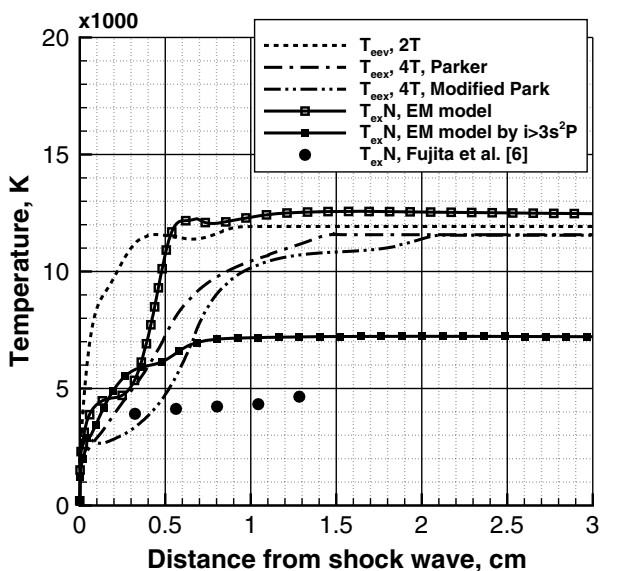

Fig. 13 Comparison of the electronic temperatures for the 2T, 4T with Parker, 4T with modified Park, and EM models in the case of the shocktube experiment by Fujita et al. [6].

relaxations affect the heavy-particle-impact dissociations, and the other chemical reactions are triggered by the dissociated $\mathrm{N}$ atoms. In Fig. $11 \mathrm{~b}$, the chemical reactions of the EM model occur more rapidly than those of the 4T with modified Park model. In the EM model, the heavy-particle-impact dissociation of each electronic state of $\mathrm{N}_{2}$ is described, and these chemical reactions produce faster dissociation than the $4 \mathrm{~T}$ with the modified Park model. The fast dissociation affects the associative ionization, electron-impact processes, and the other chemical reactions.

In Fig. 12, the computed translational, rotational, and vibrational temperatures are compared with the shock-tube experiments by Fujita et al. [6]. In the shock-tube experiments, air radiation from behind strong shock waves was measured for the $\mathrm{N}_{2}(2+)$ system using a free-piston double-diaphragm shock tube. The radiation spectra were obtained using spatially resolved imaging spectroscopy at a shock velocity of $11.9 \mathrm{~km} / \mathrm{s}$ in the ambient pressure at 0.3 torr. A series of pointwise spectroscopy analyses was carried out in order to obtain a spatial profile of temperatures. In the post normal shock flow calculations, the freestream conditions are set to the shock-tube experimental conditions. In Figs. 12a $-12 \mathrm{c}$, the rotational relaxation of the $4 \mathrm{~T}$ with Parker model is more rapidly converged to the translational temperature than the vibrational temperature. In the comparisons of the rotational and vibrational temperatures between the $2 \mathrm{~T}$ and $4 \mathrm{~T}$ with Parker models and the experimental values, it is shown that the calculated vibrational temperatures overestimate the measured temperatures, and the calculated rotational temperature of the 4T with Parker model has a discernable difference with the measured rotational values. In the rotational and vibrational temperatures of the $4 \mathrm{~T}$ with modified Park and EM models, the relaxations occur more slowly than those of the 2T and 4T with Parker models, and the calculated rotational and vibrational temperatures fall within the error bars of the measured rotational and vibrational temperatures. These results show that strong rotational and vibrational nonequilibrium exists behind the strong shock wave, and the 4T with modified Park and EM models can capture this phenomenon.

In Fig. 13, the calculated electronic temperatures by the 2T, 4T with Parker, 4T with modified Park, and EM models are compared with the measured electronic temperature of the $\mathrm{N}$ atom. The measured electronic temperature of $\mathrm{N}$ is estimated by the spectral matching method for the wavelength of $380-440 \mathrm{~nm}$. In this wavelength, the radiative transitions of $3 s^{2} P-3 p^{4} D, 3 s^{2} P-5 p^{2} D$, $3 s^{2} P-3 p^{2} D$, and $3 s^{4} P-4 p^{4} S$ are dominant. In the EM model, the electronic temperature of $\mathrm{N}$ is evaluated using the electronic excited states above $3 s^{2} P$ and compared with the experimental values. As shown in Fig. 13, such electronic temperatures of $\mathrm{N}$ do not accurately reproduce the experimental values. In this shock-tube experiment, the translational temperature immediately behind the shock is about $90,000 \mathrm{~K}$, and this temperature is outside the range of the existing transition rate data. The existing heavy-particle-impact transition rates are mostly extrapolated from rates at temperatures under $3,000 \mathrm{~K}$. This limitation may account for the difficulty in accurately reproducing the measured electronic temperature. However, the electronic temperature of atomic $\mathrm{N}$ predicted by the EM model is closer to the experimental values the results obtained with the other 2T, 4T with Parker, and 4T with modified Park models.

In Fig. 14, comparisons of the species mole fractions for the 2T, 4T, and EM models are presented. In Figs. $14 \mathrm{a}$ and $14 \mathrm{~b}$, it is shown that the chemical reactions by the $4 \mathrm{~T}$ with modified Park and EM models occur more slowly than those of the $2 \mathrm{~T}$ and $4 \mathrm{~T}$ with Parker models. These patterns of the chemical reactions are observed in Figs. 7 and 11. Unlike the chemical reactions in the above post normal shock flow cases, the electron-impact ionization and radiative recombination are dominant chemical reactions in the downstream. In the EM model, such electron-impact ionization and radiative recombination are accurately described by considering the chemical reactions of each electronic state.

In Fig. 15, the species mole fractions and temperatures for optically thin and thick media are compared in the case of the shock-tube experiments by Fujita et al. [6]. In Figs. 15a and 15b, the mole fractions and the electron number density obtained with each radiative escape factor are presented. Immediately behind the shock wave, there is no obvious effect of the radiative escape factor. However, in the downstream region, the escape factor has an important role in radiative and dielectric recombination. The electrons produced by electron-impact ionization are recombined by the radiative processes, and the electron number density in the optically thick medium is about two times larger than that in the optically thin medium. The temperature differences between the optically thin and thick media are shown in the profiles of translational, rotational, and

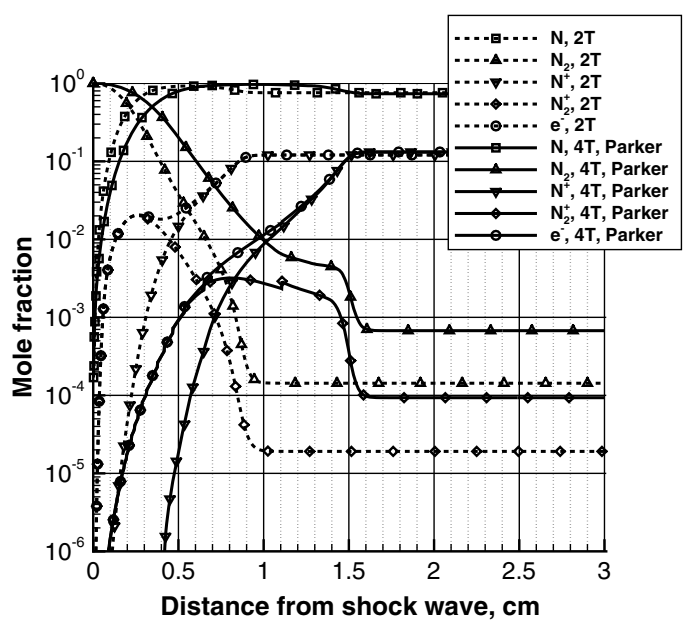

a) $2 \mathrm{~T}$ and $4 \mathrm{~T}$ with Parker models

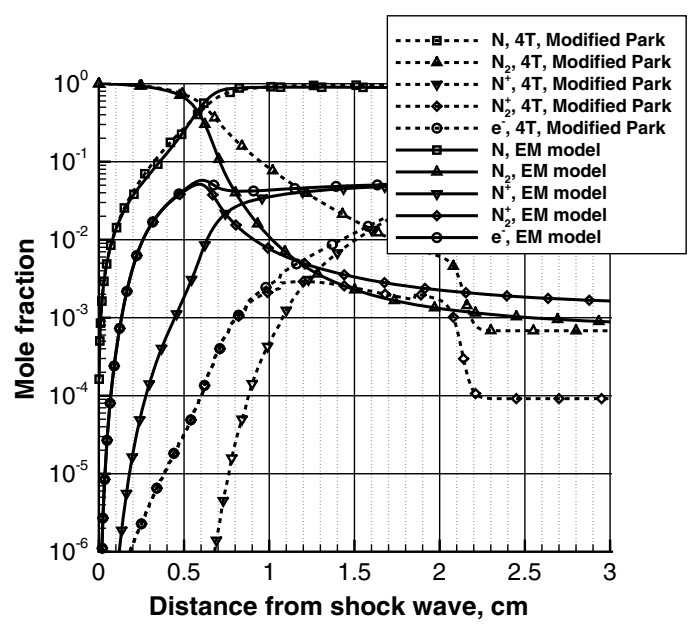

b) $4 \mathrm{~T}$ with modified Park and EM models

Fig. 14 Comparisons of the species mole fractions in the case of the shock-tube experiment by Fujita et al. [6]. 


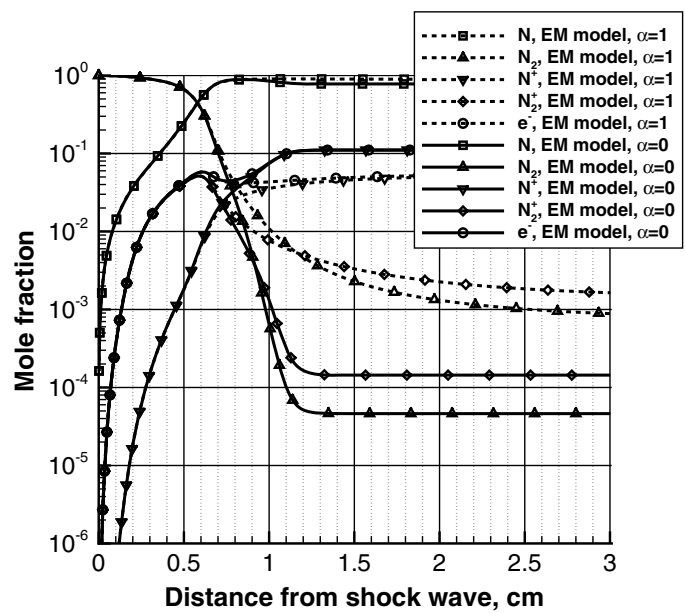

a) Species mole fractions

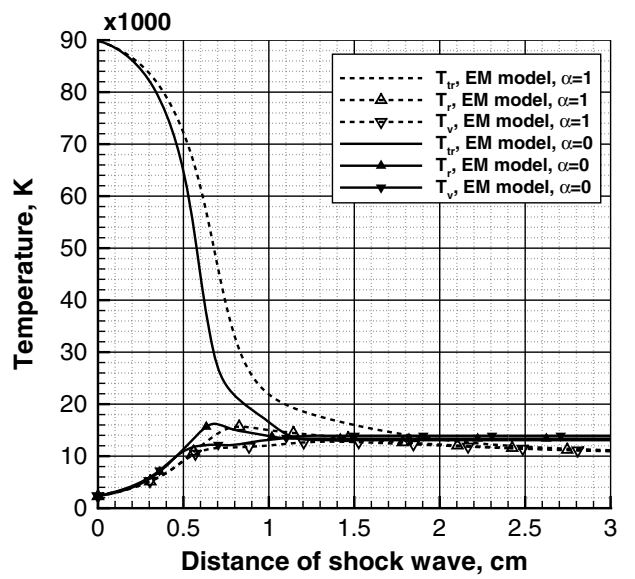

c) Translational, rotational, and vibrational temperatures

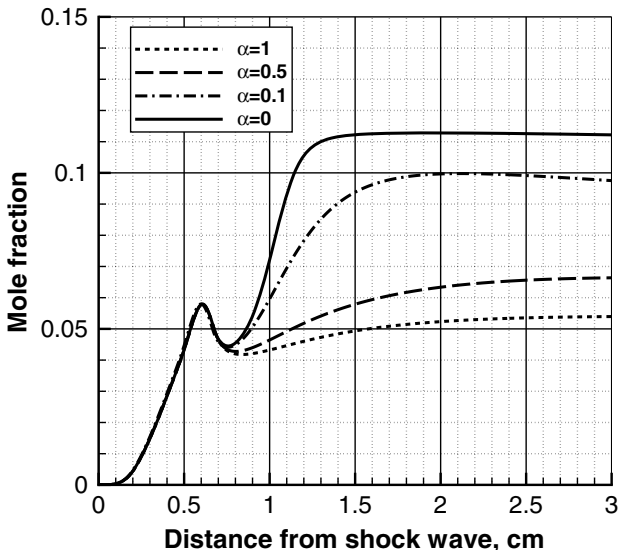

b) Mole fraction of electron

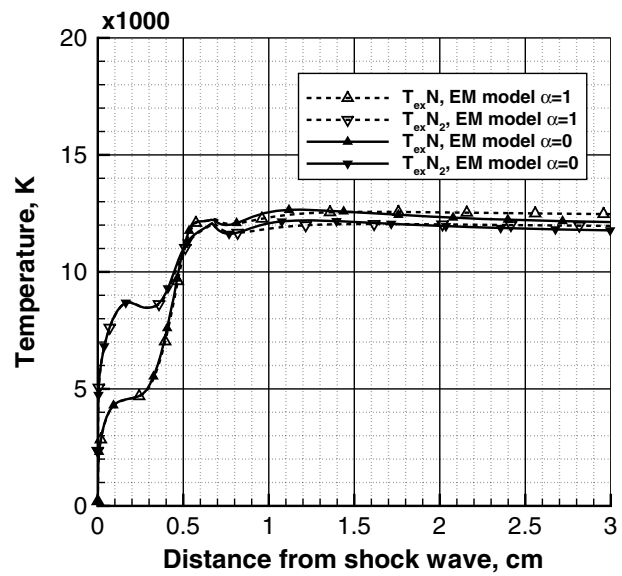

d) Species electronic temperatures

Fig. 15 Comparisons of the mole fractions and temperatures for optically thin and thick media in the case of the shock-tube experiment by Fujita et al. [ㅁ]

vibrational temperatures of Fig. $15 \mathrm{c}$. In the optically thin medium, the rovibrational relaxation occurs more slowly than that in the optically thick medium because the radiative recombined number density of $\mathrm{N}_{2}$ in the optically thin medium is larger than that of the optically thick medium. However, in the profiles of the species electronic temperatures of Fig. 15d, the differences of the temperatures between the optically thin and thick media are negligible because the species electronic excitation is dominated by the heavy-particle- and electron-impact processes.

\section{Conclusions}

In the present work, one-dimensional post normal shock flow calculations of $\mathrm{N}_{2}$ are carried out in order to analyze existing shocktube experiments. State-of-the-art thermochemical nonequilibrium models of the two-temperature (2T), four-temperature (4T), and electronic master equation coupling (EM) models are adopted in the present work. In the 4T model, the rotational nonequilibrium is described by Parker and modified Park models. In the EM model, the system of electronic master equations is constructed by the recently evaluated electron and heavy-particle impacts and radiative transition cross-sections. In the 4T with modified Park and EM models, the rotational and vibrational relaxations are slow enough to be treated as a nonequilibrium state, and the calculated temperatures agree with the shock-tube measured data. However, the rotational relaxation of the 4T with Parker model is much faster than the 4T with modified Park and EM models. These rotational and vibrational relaxations affect the heavy-particle-impact dissociation behind the shock wave. As a result of these relaxations, the chemical reactions of the 4T with modified Park and EM models occur more slowly than the chemical reactions of the $2 \mathrm{~T}$ and $4 \mathrm{~T}$ with Parker models. Immediately behind the shock wave, the electronic states are mostly excited by the heavyparticle-impact processes, and the measured electronic temperatures at the intermediate shock speed of $6 \mathrm{~km} / \mathrm{s}$ are accurately reproduced by the present EM model. However, there is a limitation to describe the excitation at a translational temperature above $90,000 \mathrm{~K}$ because this temperature is beyond the available range to describe heavyparticle-impact processes by the existing transition rate data. In the chemical reactions of the high-speed and high-temperature flows, the electron-impact ionization and radiative recombination are dominant chemical reactions in the downstream flows, and the radiative escape factor has an important role in describing these processes.

\section{Acknowledgments}

The authors gratefully acknowledge funding for this work through Air Force Office of Scientific Research Grants FA9550-11-1-0309 and FA9550-12-1-0483. The authors thank R. Jaffe and D. Schwenke at NASA Ames Research Center for providing the rovibrational stateto-state transition data of $\mathrm{N}+\mathrm{N}_{2}$. We also gratefully acknowledge $\mathrm{C}$. Park and M. Panesi for useful discussion on the electron- and heavyparticle-impact excitation processes.

\section{References}

[1] Park, C., Nonequilibrium Hypersonic Aerothermodynamics, Wiley, New York, 1990, pp. 119-144.

[2] Gupta, R. N., Moss, J. N., and Price, J. M., "Assessment of Thermochemical Nonequilibrium and Slip Effects for Orbital Reentry Experiment (OREX)," NASA TM-111600, July 1996.

[3] Furudate, M., Nonaka, S., and Swada, K., "Calculation of Shock Shapes over Simple Geometries in Intermediate Hypersonic Air Flow," 33rd AIAA Thermophysics Conference, AIAA Paper 1999-3686, June 1999. 
[4] Suzuki, K., Kubota, H., Fujita, K., and Abe, T., "Chemical Nonequilibrium Ablation Analysis of MUSES-C Super-Orbital Reentry Capsule," 32nd AIAA Thermophysics Conference, AIAA Paper 19972481, June 1997.

-[5] Sharma, S. P., and Gillespie, W., "Nonequilibrium and Equilibrium Shock Front Radiation Measurements," Journal of Thermophysics and Heat Transfer, Vol. 5, No. 3, 1991, pp. 257-265. doi: $10.2514 / 3.259$

-[6] Fujita, K., Sato, S., Abe, T., and Ebinuma, Y., "Experimental Investigation of Air Radiation Behind a Strong Shock Wave," Journal of Thermophysics and Heat Transfer, Vol. 16, No. 1, 2002, pp. 77-82. doi: $10.2514 / 2.6654$

-[7] Sakurai, K., Bindu, V. H., Niinomi, S., Ota, M., and Maeno, K., "CARS Measurement of Vibrational/Rotational Temperatures with Total Radiation Visualization Behind Strong Shock Waves of 5-7 km/s," 27th International Symposium on Rarefied Gas Dynamics, AIP Conference Proceedings 1333, AIP Publ., Melville, NY, 2011, pp. 419-424.

- [8] Matsuda, A., Ota, M., Arimura, K., Bater, S., Maeno, K., and Abe, T., "Assessment of Rotational and Vibrational Temperatures Behind Strong Shock Waves Derived from CARS Method," 26th International Symposium on Shock Waves, Vol. 1, Springer, Berlin, 2009, pp. 433-438.

-[9] Kim, J. G., and Boyd, I. D., "State-Resolved Master Equation Analysis of Thermochemical Nonequilibrium of Nitrogen," Chemical Physics, Vol. 415, No. 3, 2013, pp. 237-246. doi:10.1016/j.chemphys.2013.01.027

[10] Jaffe, R., Schwenke, D., Chaban, G., and Huo, W., "Vibrational and Rotational Excitation and Relaxation of Nitrogen from Accurate Theoretical Calculation," 46th AIAA Aerospace Sciences Meeting and Exhibit, AIAA Paper 2008-1208, Jan. 2008.

[11] Jaffe, R., Schwenke, D., and Chaban, G., "Theoretical Analysis of " $N$, "2 Collisional Dissociation and Rotation-Vibration Energy Transfer," 47th AIAA Aerospace Sciences Meeting and Exhibit, AIAA Paper 20091569, Jan. 2009.

[12] Parker, J. G., "Rotational and Vibrational Relaxation in Diatomic Gases," Physics of Fluids, Vol. 2, No. 4, 1959, pp. 449-462. doi:10.1063/1.1724417

[13] Park, C., "Rotational Relaxation of N2 Behind a Strong Shock Wave," Journal of Thermophysics and Heat Transfer, Vol. 18, No. 4, 2004, pp. 527-533. doi:10.2514/1.11442

[14] Hyun, S. Y., "Radiation Code SPRADIAN07 and Its Applications," Ph.D. Dissertation, Dept. of Aerospace Engineering, Korea Advanced Inst. of Science and Technology, Daejeon, Republic of Korea, 2009.

[15] Park, C., Jaffe, R., and Partridge, H., "Chemical-Kinetic Parameters of Hyperbolic Earth Entry," Journal of Thermophysics and Heat Transfer, Vol. 15, No. 1, 2001, pp. 76-90. doi: $10.2514 / 2.6582$

[16] Kim, J. G., and Boyd, I. D., "State-Resolved Thermochemical Nonequilibrium Analysis of Hydrogen Mixture Flows," Physics of Fluids, Vol. 24, No. 8, 2012, Paper 086102. doi:10.1063/1.4747340

[17] Gnoffo, P. A., Gupta, R. N., and Shinn, J. L., "Conservation Equations and Physical Models for Hypersonic Air Flows in Thermal and Chemical Nonequilibrium," NASA TP-2867, Feb. 1989.
[18] Nishida, M., and Matsumoto, M., "Thermochemical Nonequilibrium in Rapidly Expanding Flows of High-Temperature Air," Zeitschrift fur Naturforschung, Teil A. Physik, Physikalische Chemie, Kosmophysik, Vol. 52, No. 4, 1997, pp. 358-368.

[19] Millikan, R. C., and White, D. R., "Systematics of Vibrational Relaxation," Journal of Chemical Physics, Vol. 39, No. 12, 1963, pp. 32093213.

doi: $10.1063 / 1.1734182$

[20] Park, C., "Review of Chemical-Kinetic Problems of Future NASA Mission, I: Earth Entries," Journal of Thermophysics and Heat Transfer, Vol. 7, No. 3, 1993, pp. 385-398. doi: $10.2514 / 3.431$

[21] Lee, J. H., "Electron-Impact Vibrational Relaxation in HighTemperature Nitrogen," Journal of Thermophysics and Heat Transfer, Vol. 7, No. 3, 1993, pp. 399-405. doi:10.2514/3.432

[22] Kim, J. G., Kwon, O. J., and Park, C., "Master Equation Study and Nonequilibrium Chemical Reactions for $\mathrm{H}+\mathrm{H}_{2}$ and $\mathrm{He}+\mathrm{H}_{2}$," Journal of Thermophysics and Heat Transfer, Vol. 23, No. 3, 2009, pp. 443-453. doi:10.2514/1.41741

[23] Kim, J. G., Kwon, O. J., and Park, C., "Master Equation Study and Nonequilibrium Chemical Reactions for Hydrogen Molecule," Journal of Thermophysics and Heat Transfer, Vol. 24, No. 2, 2010, pp. 281-290. doi: $10.2514 / 1.45283$

[24] Bultel, A., Cheron, B., Boudon, A., Motapon, O., and Schneider, I., "Collisional-Radiative Model in Air for Earth Re-Entry Problems," Physics of Plasmas, Vol. 13, No. 4, 2006, pp. 1-11.

[25] Panesi, M., Magin, T. E., Bourdon, A., Bultel, A., and Chazot, O., "Fire II Flight Experiment Analysis by Means of a Collisional-Radiative Model," Journal of Thermophysics and Heat Transfer, Vol. 23, No. 2, 2009, pp. 236-248. doi:10.2514/1.39034

[26] Panesi, M., Magin, T. E., Bourdon, A., Bultel, A., and Chazot, O., "Electronic Excitation of Atoms and Molecules for the FIRE II Flight Experiment," Journal of Thermophysics and Heat Transfer, Vol. 25, No. 3, 2011, pp. 361-374. doi:10.2514/1.50033

[27] Park, C., "Rate Parameters for Electronic Excitation of Diatomic Molecules I. Electron-Impact Processes," 46th AIAA Aerospace Sciences Meeting and Exhibit, AIAA Paper 2008-1206, Jan. 2008.

[28] Park, C., "Rate Parameters for Electronic Excitation of Diatomic Molecules II. Heavy Particle-Impact Processes," 46th AIAA Aerospace Sciences Meeting and Exhibit, AIAA Paper 2008-1446, Jan. 2008.

[29] Bourdon, A., and Vervisch, P., "Three Body Recombination Rates of Atomic Nitrogen in Low Pressure Plasma Flows," Physical Review E, Vol. 54, No. 2, 1996, pp. 1888-1898. doi:10.1103/PhysRevE.54.1888

[30] Allen, R. A., "Nonequilibrium Shock Front Rotational, Vibrational, and Electronic Temperature Measurements," AVCO Everett Research Lab., Research Rept. 186, Everett, MA, Aug. 1964. 
This article has been cited by:

1. A. Lemal, K. Bando, S. Nomura, H. Takayanagi, K. Fujita. Prediction of Nonequilibrium Peak Radiation During Earth's Atmosphere Suborbital Reentry. Journal of Thermophysics and Heat Transfer, ahead of print1-5. [Citation] [Full Text] [PDF] [PDF Plus]

2. Bruno E. Lopez, Christopher O. Johnston, Marco Panesi. Improved Non-Boltzmann Modeling for Nitrogen Atoms . [Citation] [PDF] [PDF Plus]

3. Daniil A. Andrienko, Iain D. Boyd. 2016. Rovibrational energy transfer and dissociation in O $2-\mathrm{O}$ collisions. The Journal of Chemical Physics 144:10, 104301. [CrossRef]

4. Daniil Andrienko, Iain D. Boyd. High Fidelity Modeling of Thermal Relaxation and Dissociation of Oxygen . [Citation] [PDF] [PDF Plus] 